\title{
An Approach for Computing Parameters for a Lagrangian Nonlinear Maneuvering and Seakeeping Model of Submerged Vessel Motion
}

\author{
Seyong Jung ${ }^{(}$, , Member, IEEE, Stefano Brizzolara ${ }^{\circledR}$, and Craig Woolsey ${ }^{\circledR}$, Senior Member, IEEE
}

\begin{abstract}
In this study, hydrodynamic forces on a submerged vessel maneuvering near a free surface are determined using a reformulated Lagrangian nonlinear maneuvering and seakeeping model derived using Lagrangian mechanics under ideal flow assumptions. A Lagrangian mechanics maneuvering model is first reformulated to simplify the computation of parameters; then, incident wave effects are incorporated into the reformulation; finally, the parameters are computed using a medium-fidelity time-domain potential-flow panel code. Predictions from the reformulated Lagrangian nonlinear maneuvering and seakeeping model, whose parameters are computed using the methods described here, are compared with direct numerical computations in two steps for a prolate spheroid maneuvering in the longitudinal plane near the free surface. First, the hydrodynamic force and moment predicted by the model are compared with solutions from the panel code for sinusoidal motion in surge, heave, and pitch in calm water. Second, the hydrodynamic force and moment are investigated for cases where the spheroid maneuvers to approach the surface in calm water and in plane progressive waves. To conclude, a physically intuitive formulation of the Lagrangian nonlinear maneuvering and seakeeping model is presented for control applications and simulations.
\end{abstract}

Index Terms-Hydrodynamic force, Lagrangian mechanics, maneuvering and seakeeping, submerged vessel dynamics.

\begin{tabular}{ll} 
& \multicolumn{1}{c}{ NOMENCLATURE } \\
$A$ & Panel area. \\
$a$ & Body oscillation amplitude. \\
$\mathrm{AO}$ & Aegir output. \\
$\boldsymbol{b}$ & Body geometry vector. \\
$\mathcal{B}$ & Vessel body. \\
$\boldsymbol{C}_{\mathrm{b}}$ & Rigid body Coriolis and centripetal matrix. \\
$\boldsymbol{C}_{\mathrm{i}}$ & Impulsive Coriolis and centripetal matrix. \\
$\boldsymbol{C}_{\mathrm{m}}$ & Memory Coriolis and centripetal matrix. \\
$\boldsymbol{D}_{\mathrm{i}}$ & Impulsive damping matrix.
\end{tabular}

Manuscript received February 16, 2020; revised September 4, 2020; accepted January 9, 2021. The work of Seyong Jung and Craig Woolsey was supported by the Office of Naval Research under Grant N00014-14-1-0651 and Grant N00014-16-1-2749. (Corresponding author: Seyong Jung.)

Associate Editor: K. von Ellenrieder.

Seyong Jung is with the New \& Renewable Energy Laboratory, KEPCO Research Institute, Daejeon 34056, South Korea (e-mail: syjung@kepco.co.kr).

Stefano Brizzolara and Craig Woolsey are with the Kevin T. Crofton Department of Aerospace and Ocean Engineering, Virginia Tech, Blacksburg, VA 24061 USA (e-mail: stebriz@vt.edu; cwoolsey@ vt.edu).

Digital Object Identifier 10.1109/JOE.2021.3052657
$\boldsymbol{D}_{\mathrm{m}} \quad$ Memory damping matrix.

$\boldsymbol{e}_{i} \quad$ Unit vector with a 1 in the $i$ th position.

$\boldsymbol{F}_{I} \quad$ Incident wave force.

Fr Froude number.

$g \quad$ Gravitational acceleration.

$G \quad$ Transformation matrix.

$h \quad$ Impulse response function (diffraction).

$H \quad$ Wave height

$\boldsymbol{J} \quad$ Kinematic transformation matrix.

$\boldsymbol{K}_{(\cdot)} \quad$ Memory kernel matrix.

$L_{\mathrm{b}} \quad$ Spheroid body length.

$L_{D} \quad$ Distance to downstream edge.

$L_{E} \quad$ Distance to exterior edge.

$L_{U} \quad$ Distance to upstream edge.

$\mathcal{L} \quad$ Complete system Lagrangian.

LNM Lagrangian nonlinear maneuvering.

LNMS LNM and seakeeping.

$M \quad$ Pitch moment.

$M_{\mathrm{b}} \quad$ Rigid body inertia matrix.

$M_{\mathcal{B}} \quad$ Deeply submerged added mass matrix.

$\Delta M_{\mathcal{B}}$ Surface-perturbed added mass matrix.

$M_{\mathrm{i}} \quad$ Impulsive added mass matrix.

$M_{\mathrm{m}} \quad$ Memory added mass matrix.

$M_{\mathcal{S}_{0}} \quad$ Undisturbed free surface added mass matrix.

$\boldsymbol{n} \quad$ Unit normal vector.

$O \quad$ Origin of earth-fixed frame.

$O^{\prime} \quad$ Origin of body-fixed frame.

$p \quad$ Pressure.

$p_{\mathrm{a}} \quad$ Atmospheric pressure.

$\boldsymbol{q} \quad$ Set of generalized coordinates.

$Q \quad$ Set of generalized forces.

$r \quad$ Position of a point on a surface.

$\boldsymbol{r}_{\mathcal{S}_{0}} \quad$ Position of a point on $\mathcal{S}_{0}$ (relative to earth-fixed frame).

$R \quad$ Proper rotational matrix.

$\mathcal{S} \quad$ Fluid domain surface.

$\mathcal{S}_{0} \quad$ Undisturbed free surface.

$t \quad$ Time.

$t_{c} \quad$ Time constant.

$T$ Period.

$\boldsymbol{v} \quad$ Translational velocity of body.

$\boldsymbol{x}_{\mathrm{b}} \quad$ Position of body.

$X \quad$ Horizontal force.

$Z \quad$ Vertical force. 


\begin{tabular}{|c|c|}
\hline$z_{\mathrm{b}}$ & Depth of body. \\
\hline$\eta$ & Wave elevation. \\
\hline$\theta_{\mathrm{b}}$ & Pitch angle of body. \\
\hline $\boldsymbol{\theta}_{\mathrm{b}}$ & Orientation of body. \\
\hline$\lambda$ & Wave length \\
\hline$\nu$ & Set of quasivelocities. \\
\hline$\rho$ & Water density. \\
\hline$\phi$ & Velocity potential. \\
\hline$\phi_{\mathrm{D}}$ & Diffraction potential. \\
\hline$\phi_{\mathrm{i}}$ & Impulsive flow potential. \\
\hline$\phi_{\mathrm{I}}$ & Incident wave potential. \\
\hline$\phi_{\mathrm{K}}$ & Kirchhoff's potential. \\
\hline$\phi_{\mathrm{m}}$ & Memory flow potential. \\
\hline$\phi_{\mathrm{R}}$ & Radiation potential. \\
\hline$\phi_{\mathrm{s}}$ & Free surface potential. \\
\hline$\varphi$ & Normalized potential vector. \\
\hline$\varphi_{\mathrm{i}}$ & Normalized potential vector for impulsive flow. \\
\hline$\varphi_{\text {ir }}$ & Rotational component of $\varphi_{\mathrm{i}}$ \\
\hline$\varphi_{\text {it }}$ & Translational component of $\varphi_{\mathrm{i}}$ \\
\hline$\varphi_{\mathrm{K}}$ & Normalized Kirchhoff's potential vector. \\
\hline$\varphi_{\mathrm{R}}$ & Normalized radiation potential vector. \\
\hline$\varphi_{\mathrm{s}}$ & Normalized free surface potential vector. \\
\hline$\varphi_{\mathcal{S}_{0} \mathrm{i}}$ & $\begin{array}{l}\text { Normalized potential vector for impulsive flow (ex- } \\
\text { pressed in earth-fixed frame). }\end{array}$ \\
\hline & Body oscillation frequency. \\
\hline & Rotational velocity of body. \\
\hline
\end{tabular}

\section{INTRODUCTION}

A COMBINED maneuvering and seakeeping model is needed to design model-based control laws for marine craft traveling in or near waves. While there is a well-developed theory associated with maneuvering and with seakeeping, individually, the body of theory that unifies the two concepts is limited in its extent and its utility because of restrictive assumptions. In this study, a Lagrangian nonlinear maneuvering (LNM) model obtained using Lagrangian mechanics for a vessel moving below a free surface [1], [2] is reformulated and extended, by incorporating wave effects, to obtain an LNM and seakeeping (LNMS) model. The reformulated LNMS model, adapted from Battista's work [1], can be used for hydrodynamic force and motion prediction for submerged vessels maneuvering in a seaway, where existing motion models would fail to capture critical nonlinear effects generated during aggressive maneuvers. Furthermore, the underlying structure of the reformulated LNMS model can be used for model-based control design to improve performance and expand the operating envelope of the vessel.

Historically, maneuvering models were developed to study course keeping and maneuvering of marine craft in otherwise undisturbed waters [3], a condition that prevails in confined regions, such as harbors, but is uncommon in the open ocean. Many of the maneuvering models were built on classical models for vessels submerged in unbounded fluid [4] by incorporating the effects of a calm free surface. Well-known maneuvering models include the ship steering equations of motion described in [5], the model for maneuvering in deep and confined waters in [6], the nonlinear rolling coupled steering model for high-speed container ships in [7] and [8], and various others presented, for example, in [9]. More recently, a first principles LNM model has been developed using Lagrangian mechanics [1], [10] for use in the design and tuning of control and estimation schemes and for efficient simulation. By following a Lagrangian modeling approach instead of the more conventional Newtonian modeling approach, one obtains the system Lagrangian that is useful for Lyapunov-based nonlinear control design, as done in [11], for example.

While early research in ship hydrodynamics was devoted primarily to maneuvering in calm water, another branch of theory was developed for scenarios where marine craft travel in a seaway at a constant forward speed (including zero speed). Early interest in seakeeping is exemplified in a paper by Weinblum and St. Denis [12], and extensive studies have been carried out since then by employing analytical [13], [14] and numerical methods [15]-[17], including strip theory codes such as OCTOPUS, PDStrip, and others; zero-speed panel methods such as WAMIT, AQWA, and MOSES [18]; and forward speed panel methods like Swan, ShipMo3D [19], and Aegir [20]. Seakeeping models can be used, for example, to assess the dynamic stability of steady, forward motion in various environmental conditions, but their use is typically restricted to simple motions such as this. The particular code chosen here, Aegir, is a high-order (B-Spline surfaces are used for panel geometry and singularity distribution), forward speed, nonlinear, time-domain, and unsteady panel method. The code has been extended with additional routines to solve for the potential flow characteristics that are needed to compute the LNMS parameters described in this article.

To overcome some of the limitations of maneuvering and seakeeping models, efforts have been made to unify the concepts for maneuvering in waves. However, these efforts have typically imposed strict conditions. For example, the conditions for slender-body theory have been assumed [14],[21], or that the ship's nominal motion is purely in surge and in a following sea [22], or that the ship moves only in the horizontal plane and does not deviate significantly from a straight course [23]. More recent and more advanced models allow small perturbations from a nominal speed and heading [9], [24]-[26].

To relax many of these assumptions, an LNMS model for a submerged vessel operating near the surface in waves was developed by Battista [2]. While submarines and underwater vehicles normally operate well below the surface, there are occasional needs to maneuver at depths shallow enough that the surface can have a significant effect on vessel motion. Battista's LNMS model can be interpreted as an extension of Kirchhoff's equations, which captures the potential flow interaction between the free surface and a submerged vessel. Like Kirchhoff's equations for the motion of a rigid body in an unbounded volume, the LNMS model omits viscous effects, propulsion, moments due to control surfaces, etc. One may approximate such effects by amending the inviscid model, as in [27], with the understanding that these approximations ignore complicated fluid/body physics that cannot be easily captured using reduced order models. On the other hand, an important feature of a well-designed model-based feedback control law is its ability to accommodate 
uncertainty in the model itself. An amended LNMS model may capture nonlinear hydrodynamic forces due to aggressive maneuvers or significant wave forcing with sufficient accuracy to allow effective nonlinear control design and stability analysis, as well as other model-based applications, such as the design of real-time training simulators.

This work describes how the components of a control-oriented reformulated LNMS model can be computed using a potential flow panel code. To elaborate, a reformulated LNMS model is derived by reformulating and incorporating incident wave effects into an LNM model for submerged vessel maneuvering [1], [10], [28]. In addition, the parameters of the reformulated LNMS model are determined using a medium-fidelity potential-flow code, and hydrodynamic forces on a submerged vessel undergoing various prescribed motions near a calm free surface and in plane progressive waves are predicted using the model, and compared with direct numerical computations. The model derivation, force prediction, and comparison process are presented as follows. First, Section II gives some brief remarks on model-based control and estimation that provide context for the article contribution. Next, in Section III, the process for reformulating the LNM model [1], [10], [28] and incorporating incident wave effects is described. In Section IV, the mediumfidelity potential-flow code that is used for parameter computation and hydrodynamic force prediction is introduced. In Section V, hydrodynamic forces are predicted for a prolate spheroid undergoing sinusoidal motion in the vertical plane using the reformulated LNMS model and compared with panel method computations. In addition, model predictions of hydrodynamic forces acting on a prolate spheroid approaching the surface in calm water and in plane progressive waves are compared with panel code solutions. In Section VI, the reformulated LNMS model is reorganized into a vector/matrix form analogous to the model formulation presented by Battista [1], resulting in a form amenable to control and estimation design. Finally, Section VII summarizes the results of this study and presents areas of further work.

\section{Remarks ON Model-BASEd CONTROL AND ESTIMATION}

This section provides a brief review of model-based control and estimation, the motivating application for the parameter computation methods developed later in this article. While the construction of a control scheme is beyond the scope, this section serves to distinguish simulation, a common objective in computing hydrodynamic effects, and the development of motion models that can be used to design effective state and disturbance estimators, feedback and feedforward control laws, and dynamically feasible trajectories to serve as control system reference commands.

Control theory is generally concerned with systems of the form

$$
\dot{\boldsymbol{x}}=\boldsymbol{f}(\boldsymbol{x}, \boldsymbol{u}, t)
$$

where $\boldsymbol{x}(t)$ is the (finite-dimensional) state, such as a submerged vessel's position and velocity, and $\boldsymbol{u}(t)$ is a user-defined input [29]. The vector field $f$ defines the system of nonlinear, ordinary differential equations that describe the state's evolution.
The dynamic equations can be amended to account for disturbance inputs $\boldsymbol{d}(t)$ and additional equations can be incorporated to account for (possibly noisy) measurements $\boldsymbol{y}(t)$ that capture aspects of the system state.

The canonical problem of control is to determine a feedback control law $\boldsymbol{u}(\boldsymbol{x}, t)$, or perhaps $\boldsymbol{u}(\boldsymbol{y}, t)$, for which the state of the true system, represented by the mathematical model earlier, converges to some desired time history $\boldsymbol{x}_{\mathrm{d}}(t)$. While there is no control design method that can address the general class of systems (1), there are well-developed methods for subclasses of these systems. The most commonly considered subclass is linear systems [30]

$$
\dot{\boldsymbol{x}}=\boldsymbol{A}(t) \boldsymbol{x}+\boldsymbol{B}(t) \boldsymbol{u} .
$$

Typically, a system model (2) is obtained from a nonlinear model (1), developed from physical principles, by linearizing about a known trajectory-usually an equilibrium or steady motion. In this case, the state vector for the system (2) represents a perturbation from the nominal state of system (1). Provided the state and control perturbations remain small, the knowledge of the physics contained in the time-varying matrices $\boldsymbol{A}(t)$ and $\boldsymbol{B}(t)$ can be used to develop state feedback control laws that perform extremely well, despite disturbances and modeling uncertainties [31]. Similarly, knowledge of the measurement process can be used to design state estimators to enable output feedback and disturbance estimators to enable active disturbance rejection [32]. But the requirement for small perturbations is restrictive. For a ship or submerged vessel maneuvering in waves, this requirement means maneuvers must be gradual enough to allow for slow updates to a seakeeping model that assumes constant speed and course.

Alternatively, a nonlinear motion model (1) may allow the use of nonlinear control design methods, such as feedback linearization [29], sliding-mode control [33], or adaptive control [34]. These and all other methods come with their own requirements on the structure of the dynamic equations, i.e., the vector field $f$ in (1).

Methods that leverage mechanical system structure are especially well suited to the control of marine craft. A Lagrangian nonlinear motion model was recently developed for a submerged vessel in waves, which presents an opportunity to develop and test new nonlinear control and estimation strategies. For this motion model, the dynamic equations defined by the vector field $f$ exhibit a particular structure (e.g., a nonlinear integrator cascade from force to velocity to position) that can be exploited in control design. Of particular values are features related to the model's mechanical system formulation, such as energy and momentum conservation laws, that can be used for Lyapunov-based control design in which one simultaneously designs the nonlinear control law and proves its effectiveness by constructing a Lyapunov function [35].

To illustrate the idea, consider a conventional (time-invariant) Lagrangian mechanical system, i.e., one for which a generalized coordinate variable $q_{i}$ is associated to each of $n$ degrees of freedom. For simplicity, we assume an input force $u_{i}$ can be chosen to affect each of these degrees of freedom independently. Given the energy like Lagrangian function $\mathcal{L}(\boldsymbol{q}, \dot{\boldsymbol{q}})$, the system 


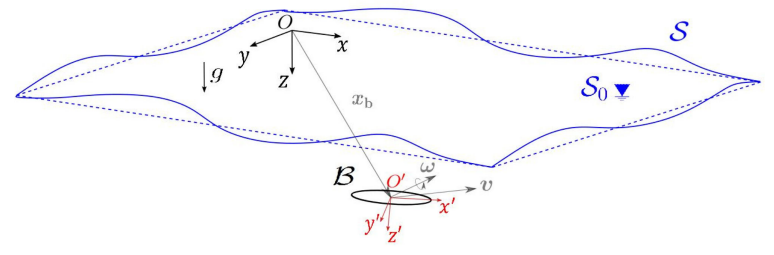

Fig. 1. Submerged vessel maneuvering near waves.

motion is described by the Euler-Lagrange equations

$$
\frac{d}{d t} \frac{\partial \mathcal{L}}{\partial \dot{\boldsymbol{q}}}-\frac{\partial \mathcal{L}}{\partial \boldsymbol{q}}=\boldsymbol{u} \Rightarrow \boldsymbol{M}(\boldsymbol{q}) \ddot{\boldsymbol{q}}+\boldsymbol{C}(\boldsymbol{q}, \dot{\boldsymbol{q}}) \dot{\boldsymbol{q}}+\boldsymbol{g}(\boldsymbol{q})=\boldsymbol{u}
$$

where $\boldsymbol{q}$ and $\boldsymbol{u}$ are the vectors of configuration and input variables, respectively, $\boldsymbol{M}(\boldsymbol{q})$ is the generalized inertia matrix, $\boldsymbol{C}(\boldsymbol{q}, \dot{\boldsymbol{q}})$ is the associated "Coriolis and centripetal" matrix, and $\boldsymbol{g}(\boldsymbol{q})$ is the generalized force due to potential energy. One approach to control design (see, for example, [35] and the references contained there) is to choose state feedback $\boldsymbol{u}(\boldsymbol{q}, \dot{\boldsymbol{q}})$ so that the closed-loop system is still Lagrangian, but with respect to a new, control-modified Lagrangian function $\mathcal{L}_{\mathrm{c}}(\boldsymbol{q}, \dot{\boldsymbol{q}})$

$$
\frac{d}{d t} \frac{\partial \mathcal{L}_{\mathrm{c}}}{\partial \dot{\boldsymbol{q}}}-\frac{\partial \mathcal{L}_{\mathrm{c}}}{\partial \boldsymbol{q}}=\mathbf{0} \Rightarrow \boldsymbol{M}_{\mathrm{c}}(\boldsymbol{q}) \ddot{\boldsymbol{q}}+\boldsymbol{C}_{\mathrm{c}}(\boldsymbol{q}, \dot{\boldsymbol{q}}) \dot{\boldsymbol{q}}+\boldsymbol{g}_{\mathrm{c}}(\boldsymbol{q})=\mathbf{0} .
$$

The associated, control-modified energy $E_{\mathrm{c}}$ is conserved by construction and serves as the basis for a Lyapunov function. Previously, unmodeled effects, such as dissipation, may be incorporated and studied using Lyapunov stability theory, which provides guidance about whether and how these additional effects should be treated. An alternative formulation can be developed for systems with symmetries of motion [36], and the concept of energy shaping extends to these systems as well, both in the Lagrangian and the Hamiltonian setting [37], [38].

The actual design of energy-based control laws is beyond the scope of this article. This article instead describes a method to compute parameter values for the control-oriented motion model. But the promise of developing more effective nonlinear control laws serves as the primary motivation for this work.

\section{LAGRANGIAN MECHANICAL SYSTEM MODEL}

This section describes the derivation process for the reformulated LNMS model using Lagrangian mechanics. Section III-A introduces the LNM model for a surface-affected submerged vessel that was developed by Battista [1] and describes the reformulation of the LNM model necessary for parameter computation. Section III-B explains how incident wave effects are incorporated into the reformulated LNM model to yield the LNMS model.

\section{A. Reformulated LNM Model}

1) LNM Model: An LNM model for a marine craft maneuvering near a calm free surface was developed from first principles by Battista [1]. Fig. 1 shows the reference frames and notation used in [1], which are also adopted here.

The system of interest is composed of a rigid body $\mathcal{B}$ moving through a semi-infinite volume of fluid with a free surface $\mathcal{S}$. The origin $O$ of the earth-fixed reference frame is fixed at the undisturbed free surface $\mathcal{S}_{0}$, whereas the origin $O^{\prime}$ of the body-fixed reference frame is fixed at the geometric center of the body $\mathcal{B}$. The $z$-axis points downward in the local direction of gravity. The generalized coordinates $\boldsymbol{q}=\left(\boldsymbol{x}_{\mathrm{b}}^{T}, \boldsymbol{\theta}_{\mathrm{b}}^{T}\right)^{T}$ describe the configuration of the body, where the vector $x_{\mathrm{b}}$ denotes the position of $O^{\prime}$ in the earth-fixed frame and the vector $\boldsymbol{\theta}_{\mathrm{b}}$ comprises the roll, pitch, and yaw Euler angles. Although this attitude parameterization suffers a singularity at pitch angles $\pm 90^{\circ}$, typical submerged vessels are unlikely to assume that orientation when operating near the surface. Alternative parameterizations are available for the rare exceptions. The quasi velocity vector $\boldsymbol{\nu}=\left(\boldsymbol{v}^{T}, \boldsymbol{\omega}^{T}\right)^{T}$ is composed of the translational velocity $\boldsymbol{v}$ and rotational velocity $\boldsymbol{\omega}$ of the body-fixed frame with respect to the earth-fixed frame, expressed in the body-fixed frame.

For simplicity, if the rigid body is assumed to be neutrally buoyant with uniformly distributed mass, the buoyant force is equal to the body weight and the centers of buoyancy and mass coincide, and therefore, the hydrostatic restoring moment may be neglected. Then, the complete rigid body and fluid system Lagrangian can be defined as

$$
\begin{aligned}
\mathcal{L}= & \frac{1}{2} \boldsymbol{\nu}^{T}\left(\boldsymbol{M}_{\mathrm{b}}+\boldsymbol{M}_{\mathcal{B}}+\Delta \boldsymbol{M}_{\mathcal{B}}+\boldsymbol{M}_{\mathcal{S}_{0}}\right) \boldsymbol{\nu} \\
& +\boldsymbol{\nu}^{T} \int_{0}^{t}\left(\boldsymbol{K}_{\mathcal{B}}+\boldsymbol{K}_{\mathcal{S}_{0}}\right) \boldsymbol{\nu}(\tau) d \tau
\end{aligned}
$$

where the matrix $\boldsymbol{M}_{\mathrm{b}}$ is the body inertia matrix, $\boldsymbol{M}_{\mathcal{B}}$ is the deeply submerged added mass matrix, $\Delta M_{\mathcal{B}}$ accounts for the asymmetry in the fluid volume due to the free surface boundary, $\boldsymbol{M}_{\mathcal{S}_{0}}$ accounts for the instantaneous deformation of the bounding surface, and the integral term accounts for memory effects. The elements of the matrices with subscripts $\mathcal{B}$ and $\mathcal{S}_{0}$ are integral functions over the rigid body and the undisturbed free surface, respectively, of the fluid velocity potential [1].

The complete system Lagrangian can then be used in the Boltzmann-Hamel equations

$$
\frac{d}{d t} \frac{\partial \mathcal{L}}{\partial \boldsymbol{\nu}}+\boldsymbol{G}(\boldsymbol{\nu}) \frac{\partial \mathcal{L}}{\partial \boldsymbol{\nu}}-\boldsymbol{J}(\boldsymbol{q})^{T} \frac{\partial \mathcal{L}}{\partial \boldsymbol{q}}=\boldsymbol{J}(\boldsymbol{q})^{T} \boldsymbol{Q}
$$

where the transformation matrices

$$
G=\left(\begin{array}{cc}
\hat{\omega} & 0 \\
\hat{v} & \hat{\omega}
\end{array}\right) \quad J=\left(\begin{array}{cc}
R & 0 \\
0 & L
\end{array}\right)
$$

are introduced due to the use of quasivelocities. The hat operator $\hat{r}$ in $G$ represents the cross product equivalent matrix of a vector. The proper rotation matrix $\boldsymbol{R}\left(\boldsymbol{\theta}_{\mathrm{b}}\right)$ in $\boldsymbol{J}$ maps free vectors from the body-fixed frame to the earth-fixed frame by a 3-2-1 sequence using the Euler angles $\boldsymbol{\theta}_{\mathrm{b}}$. The transformation matrix $\boldsymbol{L}\left(\boldsymbol{\theta}_{\mathrm{b}}\right)$ relates $\dot{\boldsymbol{\theta}}_{\mathrm{b}}$ to $\boldsymbol{\omega}$ as follows:

$$
\dot{\boldsymbol{\theta}}_{\mathrm{b}}=\boldsymbol{L}\left(\boldsymbol{\theta}_{\mathrm{b}}\right) \boldsymbol{\omega} .
$$

As a result, the matrix $\boldsymbol{J}$ expresses the following relation:

$$
\dot{\boldsymbol{q}}=\boldsymbol{J} \nu .
$$

The nonlinear Boltzmann-Hamel equation (4) describes the motion of a submerged vessel maneuvering near a calm free 
surface

$$
\begin{aligned}
\boldsymbol{F}= & \left(\boldsymbol{M}_{\mathrm{b}}+\boldsymbol{M}_{\mathrm{i}}+\boldsymbol{M}_{\mathrm{m}}\right) \dot{\boldsymbol{\nu}}+\left(\boldsymbol{C}_{\mathrm{b}}+\boldsymbol{C}_{\mathrm{i}}+\boldsymbol{C}_{\mathrm{m}}\right) \boldsymbol{\nu} \\
& +\left(\boldsymbol{D}_{\mathrm{i}}+\boldsymbol{D}_{\mathrm{m}}\right) \boldsymbol{\nu}+\int_{0}^{t} \boldsymbol{K}_{\mathrm{m}} \boldsymbol{\nu}(\tau) d \tau .
\end{aligned}
$$

The matrices with subscripts $i$ and $m$ contain the impulsive and memory flow related terms, respectively. All equations in Section III-A1 have been extracted directly from Battista's work [2] and the matrices that appear in the system Lagrangian (3), the Boltzmann-Hamel equation (4), and the equations of motion (6) are described in detail in [1] and [2].

2) Reformulation of the LNM Model: The LNM model (6) derived by Battista [1] has been reformulated for parameter computation using data from a potential flow code [10], [28]. The reformulation involves a subtle process of interchanging several integrals and differentials in the integro-differential parameters in the model, as well as determining the partial derivatives of the fluid velocity potential. The resulting reformulated LNM model (7), extracted from the work in [10], is in a form that avoids additional numerical discretization in the process already performed by the panel code, enabling higher accuracy and lower computational cost

$$
\begin{aligned}
\boldsymbol{F}= & \boldsymbol{M}_{\mathrm{b}} \dot{\boldsymbol{\nu}}+\boldsymbol{G} \boldsymbol{M}_{\mathrm{b}} \boldsymbol{\nu} \\
& +\rho \frac{d}{d t} \iint_{\mathcal{B}}(\phi \boldsymbol{b}) d S+\rho \frac{d}{d t} \iint_{\mathcal{S}_{0}}\left(\phi \frac{\partial \boldsymbol{\varphi}_{\mathrm{i}}}{\partial n}\right) d S \\
& +\rho \boldsymbol{G} \iint_{\mathcal{B}} \phi \boldsymbol{b} d S+\rho \boldsymbol{G} \iint_{\mathcal{S}_{0}} \phi \frac{\partial \boldsymbol{\varphi}_{\mathrm{i}}}{\partial n} d S \\
& -\rho \iint_{\mathcal{S}_{0}} \phi \tilde{\nabla}\left(\frac{\partial \phi}{\partial n}\right) d S+\frac{1}{2} \rho \iint_{\mathcal{S}_{0}}(\nabla \phi \cdot \nabla \phi) \boldsymbol{b} d S .
\end{aligned}
$$

Here, the body geometry vector $\boldsymbol{b}=\left(\boldsymbol{n}^{T},(\boldsymbol{r} \times \boldsymbol{n})^{T}\right)^{T}$ is composed of the unit normal vector $\boldsymbol{n}$ and the position vector $\boldsymbol{r}$ from the origin of the body-fixed frame to the point at which the normal vector $\boldsymbol{n}$ is defined. The term $\varphi_{\mathrm{i}}$ is defined as the normalized potential vector for impulsive flow, which originates from the expression $\phi=\boldsymbol{\varphi} \cdot \boldsymbol{\nu}$ for the velocity potential, proposed by Kirchhoff [4], when applied only to the impulsive component $\phi_{\mathrm{i}}$, and not the memory component $\phi_{\mathrm{m}}$, of the total velocity potential $\phi=\phi_{\mathrm{i}}+\phi_{\mathrm{m}}$. (More details on the decomposition of the total velocity potential are given in Section VI-A.) Finally

$$
\tilde{\nabla}=\left(\begin{array}{c}
\nabla \\
r \times \nabla
\end{array}\right) .
$$

The time derivative terms that appear in the second row of (7) can be replaced by equivalent expressions derived in Appendix A, where the resulting expression for the second term on the second row eventually cancels out with an another term in the model. (See Appendix B.) The resulting form is a simpler version of the reformulated LNM model

$$
\begin{aligned}
\boldsymbol{F}= & \boldsymbol{M}_{\mathrm{b}} \dot{\boldsymbol{\nu}}+\boldsymbol{G}_{\mathrm{b}} \boldsymbol{\nu}+\rho \iint_{\mathcal{B}} \frac{\partial \phi}{\partial t} \boldsymbol{b} d S+\rho \boldsymbol{G} \iint_{\mathcal{B}} \phi \boldsymbol{b} d S \\
& -\rho \iint_{\mathcal{S}_{0}} \phi \tilde{\nabla}\left(\frac{\partial \phi}{\partial n}\right) d S+\frac{1}{2} \rho \iint_{\mathcal{S}_{0}}(\nabla \phi \cdot \nabla \phi) \boldsymbol{b} d S .
\end{aligned}
$$

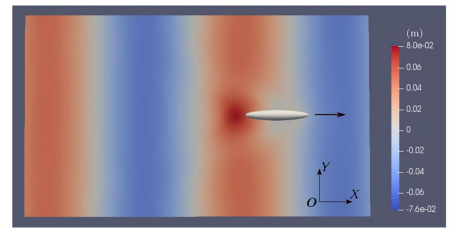

(a)

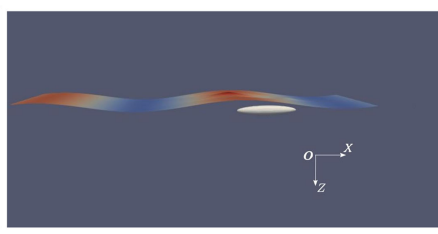

(b)

Fig. 2. Surface elevation for a horizontal, prolate spheroid approaching the free surface at constant forward speed $(\mathrm{Fr}=0.35)$. A scale factor of 3.0 was used in (b) to exaggerate the wave elevations. (a) Bottom view. (b) Side view.

\section{B. Reformulated LNMS Model}

Incident wave forces are incorporated into the reformulated LNM model (8) as an external force $\boldsymbol{F}_{I}$, which accelerates the body and counteracts the forces due to radiation and diffraction of waves. Incident wave force can be expressed using the incident wave potential $\phi_{\mathrm{I}}$ as

$$
\boldsymbol{F}_{I}=-\rho \iint_{\mathcal{B}}\left(\frac{\partial \phi_{\mathrm{I}}}{\partial t}+\frac{1}{2} \nabla \phi_{\mathrm{I}} \cdot \nabla \phi_{\mathrm{I}}\right) \boldsymbol{b} d S .
$$

Finally, the reformulated LNMS model can be written as

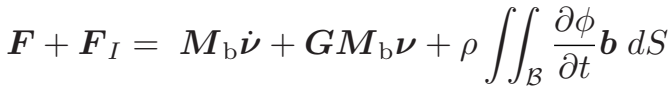

$$
\begin{aligned}
& +\rho \boldsymbol{G} \iint_{\mathcal{B}} \phi \boldsymbol{b} d S-\rho \iint_{\mathcal{S}_{0}} \phi \tilde{\nabla}\left(\frac{\partial \phi}{\partial n}\right) d S \\
& +\frac{1}{2} \rho \iint_{\mathcal{S}_{0}}(\nabla \phi \cdot \nabla \phi) \boldsymbol{b} d S
\end{aligned}
$$

where the velocity potential $\phi$ now accounts for 1) radiation waves due to body motion in the absence of incident waves and 2) diffraction waves due to scattering of the incident waves. See [28] for details.

\section{Potential-Flow Panel Code}

The velocity potential and its derivatives that appear in the reformulated LNMS model (10) can be obtained from flow solutions using the medium-fidelity potential-flow code Aegir (see Fig. 2). Aegir is a time-domain solver that uses a boundary element method for computing flow characteristics and unsteady wave patterns, as well as forces and motions of bodies moving at or near a free surface [20]. Results from Aegir have been compared against experimental data [39], and have been shown to be very accurate for certain hull forms for motion in calm water, though assessments are inconclusive for seakeeping due to limited experimental data.

Aegir, as it stands, computes $\phi_{\mathrm{I}}$ and its time derivative $\partial \phi_{\mathrm{I}} / \partial t$ in (9). Also, the code computes the memory potential $\phi$ and its time derivative $\partial \phi / \partial t$, as well as its gradient $\nabla \phi$, which appear in (10). Other values, such as the second-order partial derivative $\tilde{\nabla}(\partial \phi / \partial n)$ or the normalized potential vector for impulsive flow $\varphi_{\mathrm{i}}$, which appear in the model formulation in Section VI, require some modification of the code. While $\tilde{\nabla}(\partial \phi / \partial n)$ can be obtained with minor modifications using computations readily available from the panel code [20], [40], computing $\varphi_{\mathrm{i}}$ requires more effort. 
The components of the normalized potential vector for impulsive flow $\varphi_{\mathrm{i}}$ are not obtained from the time-domain solutions of the flow along with the other parameters of the model; they are derived separately by solving for the instantaneous response of the fluid due to an impulsive motion of the body. For example, the surge element of the normalized potential vector $\varphi_{\mathrm{i}}$ is obtained by prescribing unit velocity in surge on a body while imposing $\phi_{\mathrm{i}}=0$ at the undisturbed free surface $\mathcal{S}_{0}$, a condition derived from the linearized free surface boundary condition for impulsive motion of the body [41]. The resulting velocity potential for the instantaneous response of the fluid will be equal to the first element of the normalized potential vector for impulsive flow $\varphi_{\mathrm{i}}$. The remaining elements of the normalized potential vector can be obtained in the same manner, but by prescribing unit velocity in the corresponding direction.

The integrands of the parameters in the reformulated LNMS model (10) can be computed once the potentials $\phi_{\mathrm{I}}$ and $\phi$, their derivatives, and the normalized potential vector for impulsive flow $\varphi_{\mathrm{i}}$ are obtained. Through additional modification of the potential flow code, the individual parameters and hydrodynamic force components on the body can be obtained by integrating the computed terms over the body $\mathcal{B}$ and the undisturbed free surface $\mathcal{S}_{0}$.

\section{COMPUTATION OF HydRODYNAMIC FORCES}

Although computing each of the parameters of the reformulated LNMS model (10) individually and analyzing their sensitivity and uncertainty would be extremely informative, especially when considering control design applications, doing so would be impractical, given the extensive coupling among model terms. Therefore, in this section, hydrodynamic forces predicted using the reformulated LNMS model (10), whose parameters are computed using the methods described in Sections IV-VI, are compared with solutions from the panel code Aegir (see Section IV). In Section V-A, the force and moment acting on a prolate spheroid are compared for sinusoidal surge, heave, and pitch motion in calm water. In Section V-B, we consider the same prolate spheroid approaching the surface in calm water and in plane progressive waves, to study incident wave effects. In this scenario, the spheroid begins and ends its maneuver in steady translation parallel to the nominal free surface.

\section{A. Forces on Body Due to Sinusoidal Motion in Calm Water}

The hydrodynamic force and moment acting in the vertical plane of the earth-fixed reference frame are computed numerically for a 6:1 prolate spheroid for the conditions shown in Table I using Aegir. Although the commonly adopted 6:1 prolate spheroid geometry is used in this study, any other body form may be used so long as a finer mesh is applied to regions of complex geometry. The free surface dimensions are determined by the distances to the upstream, downstream, and exterior edges shown in Fig. 3. Hydrodynamic force and moment calculations are sensitive to free surface domain size and panel area. The dimensions used in this article match those determined to produce reliable results in a previous study [10], [28].

Formulas for the hydrodynamic force and moment acting in the vertical plane can be derived from the reformulated LNMS
TABLE I

INPUT VARIABLES-SINUSOIDAL MOTION

\begin{tabular}{|c|c|}
\hline Variable & Value \\
\hline \hline Spheroid length-to-diameter ratio & $6: 1$ \\
\hline Spheroid body length, $L_{\mathrm{b}}$ & $2 \mathrm{~m}$ \\
\hline Fluid density, $\rho$ & $1000 \mathrm{~kg} / \mathrm{m}^{3}$ \\
\hline Depth-to-body length ratio, $z_{\mathrm{b}} / L_{\mathrm{b}}$ & 0.15 \\
\hline Motion type & Sinusoidal \\
\hline Direction of motion & $\{$ surge, heave, pitch $\}$ \\
\hline Oscillation period, $T$ & $1 \mathrm{~s}$ \\
\hline $\begin{array}{c}\text { Oscillation amplitude } \\
\text { Surge/Heave, } a / L_{\mathrm{b}} \\
\text { Pitch, } a\end{array}$ & $2.5 \mathrm{e}-2$ \\
\hline Free surface type & $8.73 \mathrm{e}-2$ rad $(5 \mathrm{deg})$ \\
\hline $\begin{array}{c}\text { Free surface dimensions } \\
\left\{L_{U}, L_{D}, L_{E}\right\}\end{array}$ & $\left\{3 L_{\mathrm{b}}, 3 L_{\mathrm{b}}, 1.5 L_{\mathrm{b}}\right\}$ \\
\hline Panel area, $A / L_{\mathrm{b}}^{2}$ & $1.25 \mathrm{e}-3$ \\
\hline
\end{tabular}

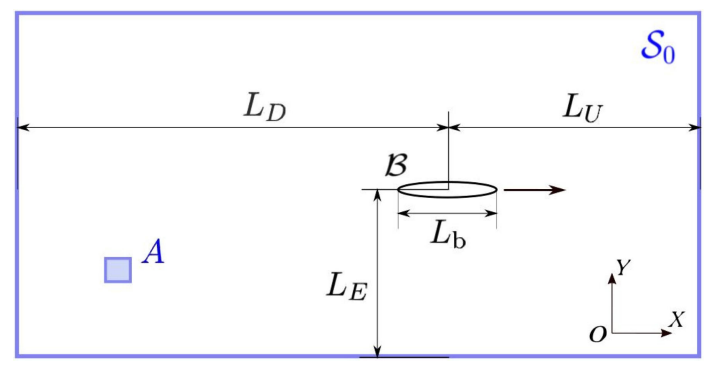

Fig. 3. Notation for submerged vessel dimensions, domain dimensions, and free surface panel area (bottom view).

model (10). In calm water, $\boldsymbol{F}_{I}$ is set to zero, and the resulting formulas for the force components in the vertical plane of the earth-fixed frame for purely surging or purely heaving motion are, respectively

$$
\begin{aligned}
X= & -\rho \iint_{\mathcal{B}} \frac{\partial \phi}{\partial t} n_{x} d S+\rho \iint_{\mathcal{S}_{0}} \phi \frac{\partial^{2} \phi}{\partial x \partial n} d S \\
& -\frac{1}{2} \rho \iint_{\mathcal{S}_{0}}(\nabla \phi \cdot \nabla \phi) n_{x} d S \\
Z= & -\rho \iint_{\mathcal{B}} \frac{\partial \phi}{\partial t} n_{z} d S+\rho \iint_{\mathcal{S}_{0}} \phi \frac{\partial^{2} \phi}{\partial n^{2}} d S \\
& -\frac{1}{2} \rho \iint_{\mathcal{S}_{0}}(\nabla \phi \cdot \nabla \phi) n_{z} d S .
\end{aligned}
$$

The formula for pitching moment in purely surging motion is

$$
\begin{aligned}
M= & -\rho \iint_{\mathcal{B}} \frac{\partial \phi}{\partial t}\left(r_{z} n_{x}-r_{x} n_{z}\right) d S+\rho \iint_{\mathcal{B}} \phi u n_{z} d S \\
& +\rho \iint_{\mathcal{S}_{0}} \phi\left(r_{z} \frac{\partial^{2} \phi}{\partial x \partial n}-r_{x} \frac{\partial^{2} \phi}{\partial n^{2}}\right) d S \\
& -\frac{1}{2} \rho \iint_{\mathcal{S}_{0}}(\nabla \phi \cdot \nabla \phi)\left(r_{z} n_{x}-r_{x} n_{z}\right) d S
\end{aligned}
$$

The formula for pitching moment in purely heaving motion is

$$
M=-\rho \iint_{\mathcal{B}} \frac{\partial \phi}{\partial t}\left(r_{z} n_{x}-r_{x} n_{z}\right) d S+\rho \iint_{\mathcal{B}} \phi w n_{x} d S
$$




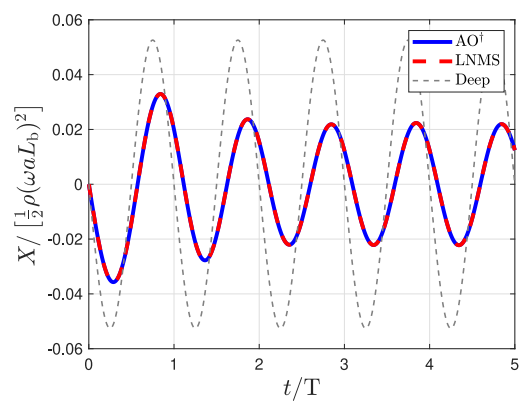

(a)

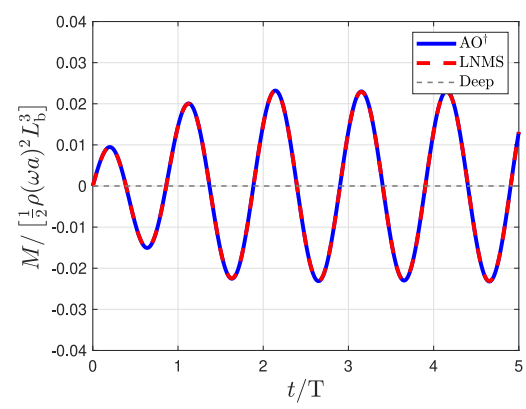

(c)

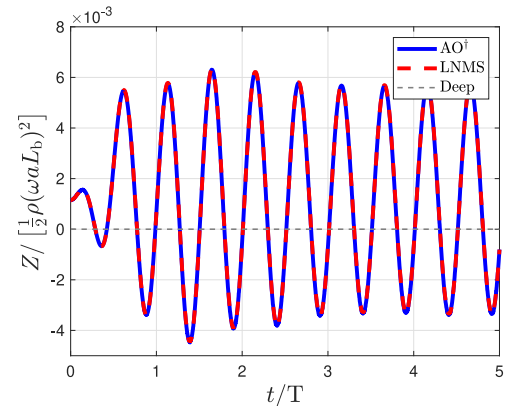

(b)

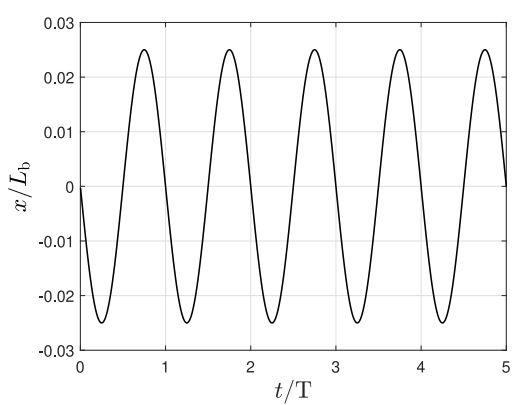

(d)

Fig. 4. Sinusoidal surging motion in calm water: (a) Horizontal force; (b) vertical force; and (c) pitch moment acting on the body; (d) corresponding position in $x$-axis. $\mathrm{AO}^{\dagger}$, Aegir Output.

$$
\begin{aligned}
& +\rho \iint_{\mathcal{S}_{0}} \phi\left(r_{z} \frac{\partial^{2} \phi}{\partial x \partial n}-r_{x} \frac{\partial^{2} \phi}{\partial n^{2}}\right) d S \\
& -\frac{1}{2} \rho \iint_{\mathcal{S}_{0}}(\nabla \phi \cdot \nabla \phi)\left(r_{z} n_{x}-r_{x} n_{z}\right) d S .
\end{aligned}
$$

The hydrodynamic force and moment components are first computed for a prolate spheroid whose longitudinal axis remains parallel to the free surface when the body undergoes pure sinusoidal surging motion with zero mean velocity while starting from rest. The results are shown in Fig. 4, where the horizontal force (along the $x$-axis in the earth-fixed reference frame), vertical force (along the $z$-axis in the earth-fixed reference frame), and pitch moment (about the $y$-axis in the earth-fixed reference frame) computations from the panel code Aegir [20] are plotted as blue solid lines, and the corresponding force and moment calculations using (11)-(13) are plotted as red dashed lines. For comparison, the hydrodynamic force and moment on a prolate spheroid undergoing the same motion in deep water (unbounded fluid), as computed by Aegir, are shown in gray dashed lines. In addition, the position of the spheroid along the horizontal axis is included in Fig. 4(d) to illustrate the frequency and phase shift between the input motion and the resulting hydrodynamic forces. It is evident that the near-surface values produced by the panel code and by the computations using the reformulated LNMS model (10) agree quite well. Note that the frequency of the horizontal force and the pitch moment oscillations matches that of the input motion, with a slight phase shift, but that the heave force frequency is double that of the input motion. Also note that transient effects are evident in the initial 2 to 3 oscillations for this constant-frequency oscillating motion because the motion starts from rest. For comparison, results for the deeply submerged case in Fig. 4(a) show that horizontal force is in-phase with position; the force decreases and the phase shifts when near the free surface. Fig. 4(b) and (c) shows that the vertical force and pitch moment are zero, for the deeply submerged body, a consequence of geometrical symmetry. It is also interesting to note that although one expects an upward force-the free surface suction-during a steady horizontal motion near the free surface, sinusoidal surging motion, as shown in Fig. 4(b), generates a mean downward force. This is because, while a low-pressure region that covers most of the mid and aft upper part of the spheroid is formed under the trough of the wave immediately downstream of the Bernoulli hump for a steady horizontal motion, this low-pressure region does not form during an unsteady sinusoidal surging motion, and a mean downward force is generated for the particular conditions.

Next, we consider the case where the body undergoes a purely sinusoidal heaving motion with zero mean velocity. The hydrodynamic force and moment are computed using the formulas in (11), (12), and (14). Fig. 5 shows that the vertical force produced by Aegir, plotted as a blue solid line, agrees very well with computations using (12), plotted as a red dashed line. The frequency of oscillation matches, with a slight phase shift between the input motion and the corresponding hydrodynamic force. For comparison, the hydrodynamic force and moment on a prolate spheroid undergoing the same motion in deep water, as computed by the panel code Aegir, are shown in gray dashed lines. The vertical force is in-phase with position, in this case, but it decreases in amplitude and the phase shifts when near the free surface. The computed values of horizontal force and pitching moment, which are not included in the figure, are 


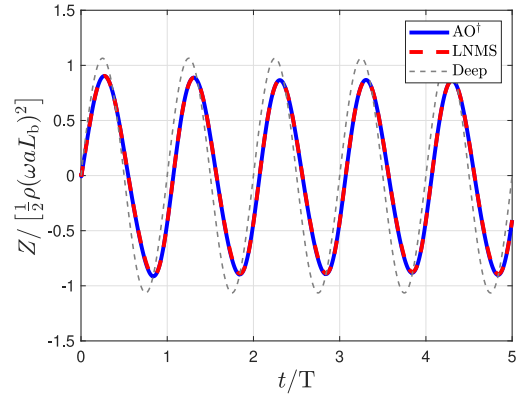

(a)

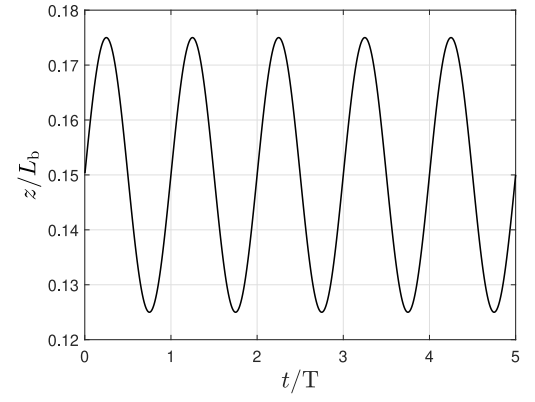

(b)

Fig. 5. Sinusoidal heaving motion in calm water. (a) Vertical force acting on the body and (b) corresponding position in the $z$-axis. AO ${ }^{\dagger}$, Aegir Output.

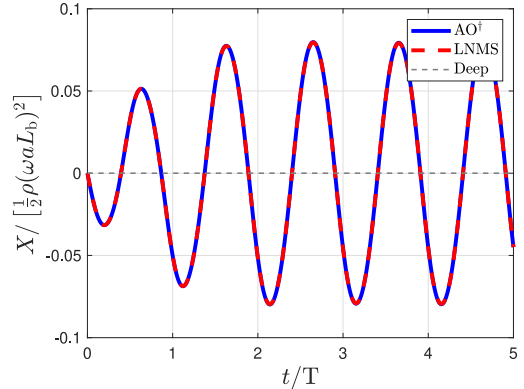

(a)

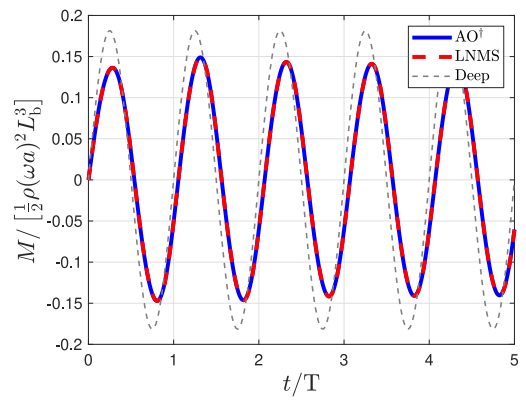

(c)

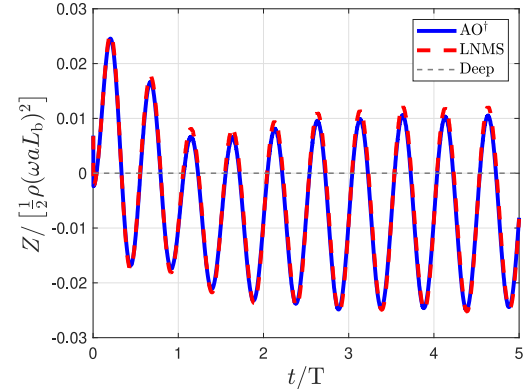

(b)

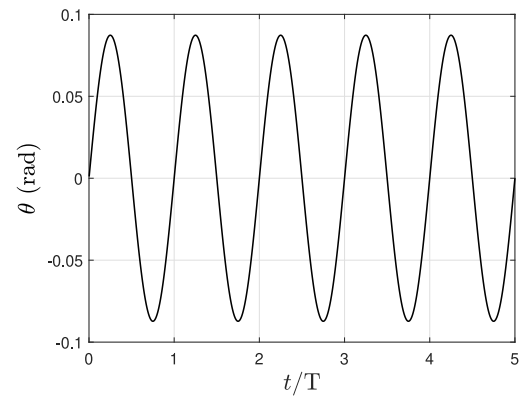

(d)

Fig. 6. Sinusoidal pitching motion in calm water: (a) Horizontal force; (b) vertical force; and (c) pitch moment acting on the body; (d) corresponding position in $y$-axis. $\mathrm{AO}^{\dagger}$, Aegir Output.

approximately 10 orders of magnitude smaller than the vertical force and decrease further with decreasing free surface panel areas. The horizontal force and pitch moment can be regarded as zero, which is consistent with expectations for a fore-aft symmetric spheroid undergoing pure heaving motion in calm water.

Moving on to purely pitching motion with zero mean velocity, formulas for the hydrodynamic force and moment in the vertical plane derived from the reformulated LNMS model (10) are shown in the following equations:

$$
\begin{aligned}
X= & -\rho \iint_{\mathcal{B}} \frac{\partial \phi}{\partial t} n_{x} d S-\rho \iint_{\mathcal{B}} \phi q n_{z} d S \\
& +\rho \iint_{\mathcal{S}_{0}} \phi \frac{\partial^{2} \phi}{\partial x \partial n} d S
\end{aligned}
$$

$$
\begin{aligned}
& -\frac{1}{2} \rho \iint_{\mathcal{S}_{0}}(\nabla \phi \cdot \nabla \phi) n_{x} d S \\
Z=- & \rho \iint_{\mathcal{B}} \frac{\partial \phi}{\partial t} n_{z} d S+\rho \iint_{\mathcal{B}} \phi q n_{x} d S \\
& +\rho \iint_{\mathcal{S}_{0}} \phi \frac{\partial^{2} \phi}{\partial n^{2}} d S-\frac{1}{2} \rho \iint_{\mathcal{S}_{0}}(\nabla \phi \cdot \nabla \phi) n_{z} d S \\
M= & -\rho \iint_{\mathcal{B}} \frac{\partial \phi}{\partial t}\left(r_{z} n_{x}-r_{x} n_{z}\right) d S \\
& +\rho \iint_{\mathcal{S}_{0}} \phi\left(r_{z} \frac{\partial^{2} \phi}{\partial x \partial n}-r_{x} \frac{\partial^{2} \phi}{\partial n^{2}}\right) d S \\
& -\frac{1}{2} \rho \iint_{\mathcal{S}_{0}}(\nabla \phi \cdot \nabla \phi)\left(r_{z} n_{x}-r_{x} n_{z}\right) d S .
\end{aligned}
$$

Fig. 6 shows the force and moment produced by Aegir, plotted in blue solid lines, and hydrodynamic force computations using 


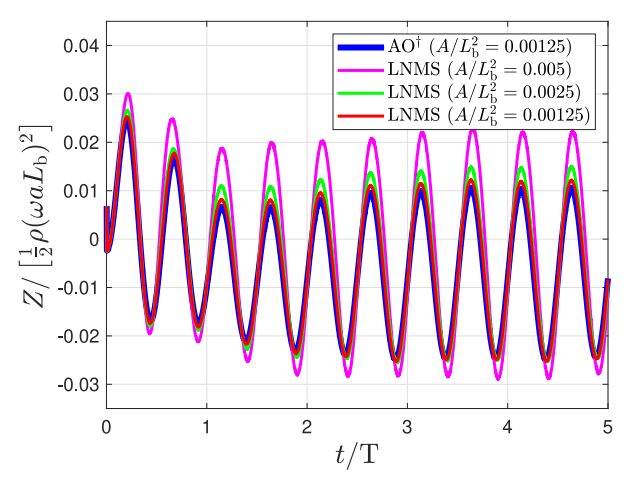

Fig. 7. Comparison of vertical force for sinusoidal pitching motion in calm water, computed using various panel areas. $\mathrm{AO}^{\dagger}$, Aegir Output.

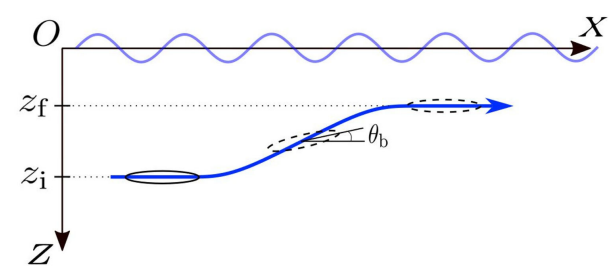

Fig. 8. Prolate spheroid approaching the surface under waves. (Axes not to scale.)

(15)-(17), plotted as red dashed lines. In addition, the hydrodynamic force and moment on a prolate spheroid undergoing the same motion in deep water, as computed by Aegir, are shown in gray dashed lines for comparison. Near-surface horizontal force and pitch moment comparisons agree very well, though some discrepancy can be seen in the vertical force. As shown in Fig. 7, the discrepancy in vertical force is mainly due to a large panel area on the free surface. It is evident that the vertical force due to purely sinusoidal pitching motion derived from (16) approaches the panel code results as panel area is decreased. However, panel area should be determined carefully according to the required accuracy of the model, recognizing that smaller panel areas increase accuracy but at the higher computational cost. Note that the mean vertical force in Fig. 6(b) adjusts over a couple of cycles, because the simulation starts from rest, before the vertical force converges to a steady-state variation. Also note the shifted steady mean value of vertical force due to the top-bottom asymmetry in free surface effects. Results for the deeply submerged case in Fig. 6(c) show that pitch moment is in-phase with angular position, and that the moment amplitude decreases and the phase shifts when near the free surface. Fig. 6(a) and (b) shows that the horizontal and vertical forces are zero, a consequence of geometrical symmetry.

\section{B. Forces on a Body Approaching the Surface}

In this section, a $6: 1$ prolate spheroid undergoing a nearsurface longitudinal maneuver (see Fig. 8) has been simulated under the conditions specified in Table II. The prolate spheroid starts from rest at depth $z_{\mathrm{i}}$ with its longitudinal axis parallel to the free surface, begins moving horizontally at constant speed and, then, begins pitching nose up and ascending at constant
TABLE II

INPUT VARIABLES-SURFACE APPROACH MANEUVER

\begin{tabular}{|c|c|}
\hline Variable & Value \\
\hline \hline Spheroid length-to-diameter ratio & $6: 1$ \\
\hline Spheroid body length, $L_{\mathrm{b}}$ & $2 \mathrm{~m}$ \\
\hline Fluid density, $\rho$ & $1000 \mathrm{~kg} / \mathrm{m}^{3}$ \\
\hline Motion type & Surface approach (Fig. 9) \\
\hline Depth-to-body length ratio & $z_{\mathrm{i}} / L_{\mathrm{b}}: 0.4$ (initial depth) \\
$z_{\mathrm{f}} / L_{\mathrm{b}}: 0.2$ (final depth)
\end{tabular}
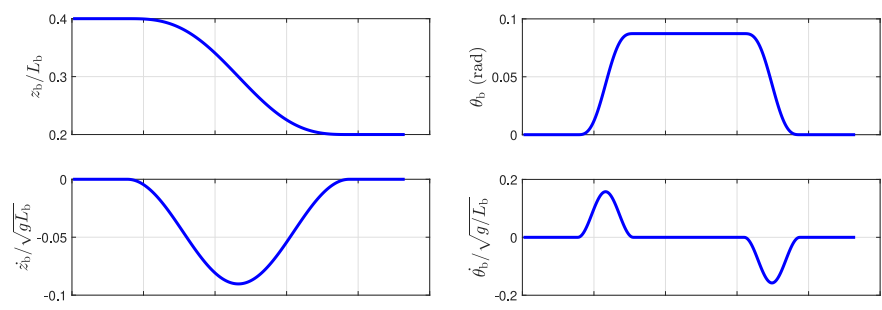

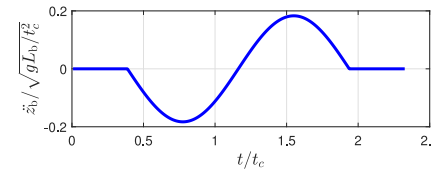

(a)

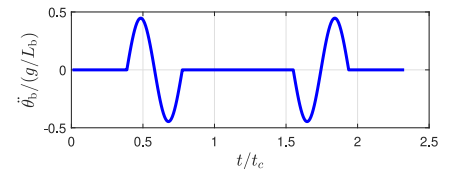

(b)
Fig. 9. Motion profile: (a) Translational and (b) rotational position, velocity, and acceleration along $z$-axis and about $y$-axis respectively, during surface approach maneuver.

speed while continuing at the given constant horizontal speed. When the spheroid approaches the shallower depth $z_{\mathrm{f}}$, it begins pitching down until the longitudinal axis is again parallel to the free surface and the vertical motion is arrested so that the spheroid once again travels horizontally at constant speed and depth. Horizontal speed is constant throughout the surface approach maneuver while the vertical and pitch motion profiles are shown in Fig. 9(a) and (b), respectively. In Fig. 9, $z_{\mathrm{b}}$ and $\theta_{\mathrm{b}}$ are depth and pitch angle of the spheroid, respectively. Also, the simulation is carried out in calm water and, then, under plane progressive waves.

Remark: The prescribed motion was constructed to mimic a near-surface maneuver; however, the given motion profile may not be the most natural for a given submerged vessel. An important application of the motion model described in this article is to generate dynamically feasible maneuvers through trajectory optimization, for example, which can be used as reference trajectories for a motion control system.

Results for a prolate spheroid approaching an otherwise undisturbed free surface are shown in Fig. 10, where values output by Aegir are plotted as blue solid lines and the corresponding force and moment calculations using (10) are plotted as red dashed 


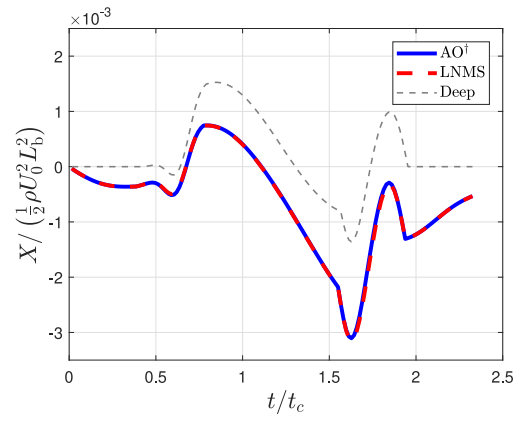

(a)

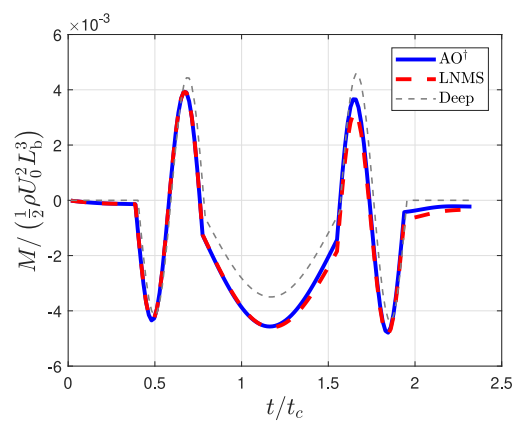

(c)

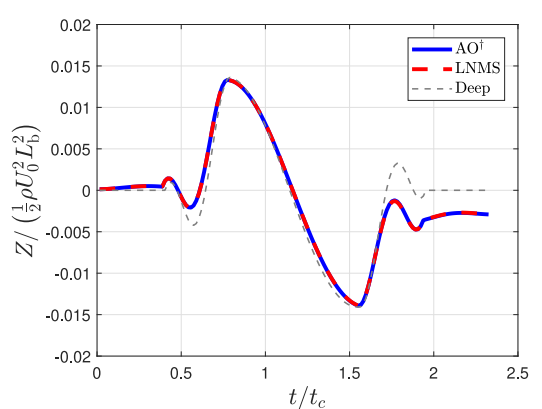

(b)

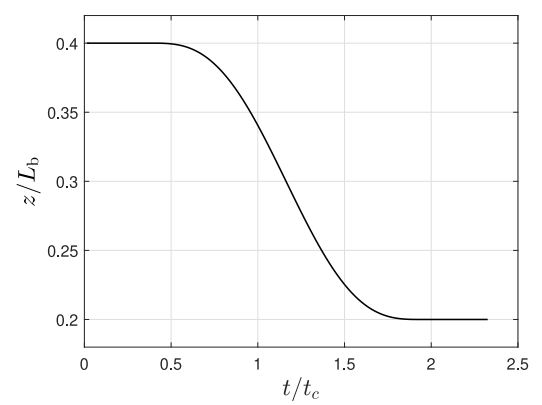

(d)

Fig. 10. Surface approach maneuver in calm water at $\mathrm{Fr}=0.35$ : (a) Horizontal force; (b) vertical force; and (c) pitch moment acting on the body; (d) corresponding position in the $z$-axis (on the horizontal axis of the plots, time constant $t_{c}=L_{\mathrm{b}} / U_{0}$ ). AO ${ }^{\dagger}$, Aegir Output.

lines. In addition, the hydrodynamic force and moment on a prolate spheroid undergoing the same motion in deep water, as computed by Aegir, are shown in gray dashed lines for comparison. Near-surface horizontal and vertical force comparisons agree very well. However, there is some discrepancy in pitch moment, which increases as the spheroid approaches the free surface. This discrepancy can be reduced by refining the panels on the free surface and increasing the overall free surface size, but at increased computational cost. Note that vertical force [see Fig. 10(b)] and pitch moment [see Fig. 10(c)] are similar in form, but opposite in sign, to the acceleration in the vertical direction [see Fig. 9(a)] and acceleration in pitch [see Fig. 9(b)], respectively. Furthermore, results for the deeply submerged case in Fig. 10(a) show the horizontal force is zero during the beginning and ending of the motion where vertical and pitching velocities are zero and horizontal velocity is constant.

Moving on to results for a prolate spheroid approaching the surface under plane progressive waves, hydrodynamic force values output by Aegir are plotted as blue solid lines and the corresponding force and moment calculated using (10) are plotted as red dashed lines in Fig. 11. Similar to the results in calm water (see Fig. 10), horizontal and vertical force comparisons agree very well while there is some discrepancy in pitch moment that increases as the spheroid approaches the free surface. The force and moment acting on the body due to the incident wave are calculated using (9) and included in Fig. 11 as solid green lines. The instant when the crest of the incident wave coincides with the geometric center of the body, is denoted by vertical, dotted gray lines. The peak in the horizontal incident wave force lags the incident wave crest [see Fig. 11(a)]; however, the phase shift appears inconsistent for pitch moment [see Fig. 11(c)] due to the antisymmetric pitching motions near the beginning and end of the maneuver. The peaks of vertical incident wave force and wave height coincide [see Fig. 11(b)]. It is also evident that the horizontal force [see Fig. 11(a)] is predominantly affected by the incident waves. There is no horizontal acceleration, so the horizontal force is influenced mainly by surface effects. On the other hand, vertical force [see Fig. 11(b)] and pitch moment [see Fig. 11(c)] are affected to some extent by the incident wave at the beginning and the end of the surface approach maneuver, where there is no displacement in the vertical direction or in pitch angle. But during pitching and ascent, the vertical force and pitch moment are dominated by body motion effects; the resulting values are similar to those in Fig. 10.

\section{CONTROL-ORIENTED MOdEL}

The reformulated LNMS model in (10) can further be rewritten into a form more amenable to control design and which may afford more physical insight. In Section VI-A, the velocity potential in the reformulated LNMS model (10) is decomposed to produce partial models that are perhaps more physically intuitive. In addition, in Section VI-B, a method for approximating and replacing the convolution integrals in the memory terms is described to generate a model suitable for control design and simulation.

\section{A. Decomposition of the Velocity Potential}

The total velocity potential $\phi$ that appears in the reformulated LNMS model (10) can be decomposed into the form proposed 


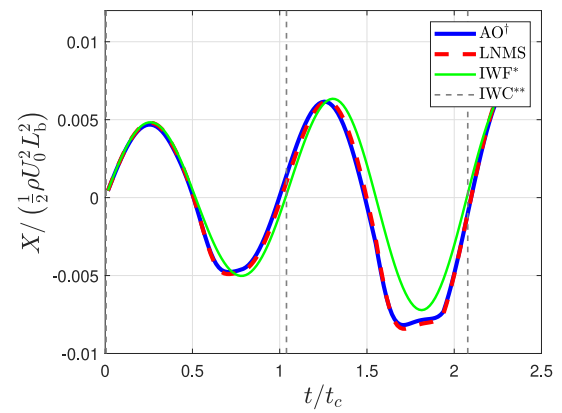

(a)

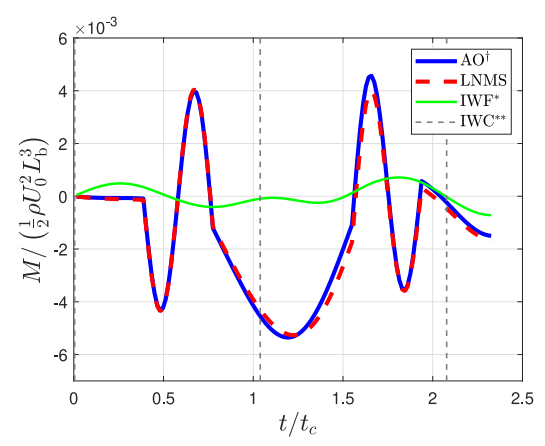

(c)

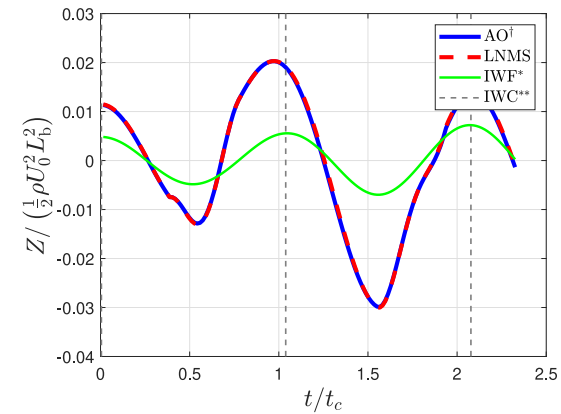

(b)

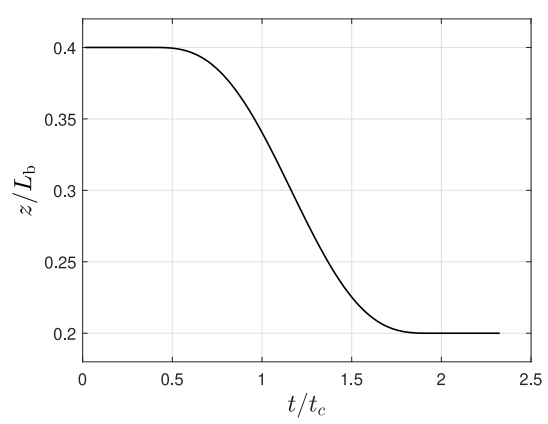

(d)

Fig. 11. Surface approach maneuver in waves $\left\langle\right.$ wavelength, $\lambda / L_{\mathrm{b}}=3.12$; wave steepness, $\left.H / \lambda=3.21 \mathrm{e}-3\right\rangle$ : (a) Horizontal force; (b) vertical force; and (c) pitch moment acting on the body; (d) corresponding position in the $z$-axis. $\mathrm{AO}^{\dagger}$, Aegir Output. IWF* Incident Wave Force. IWC ${ }^{* *}$, Incident Wave Crest.

by Battista [2]

$$
\begin{aligned}
\phi(\boldsymbol{q}, \boldsymbol{\nu}, t) & =\phi_{\mathrm{K}}(\boldsymbol{\nu})+\phi_{\mathrm{s}}(\boldsymbol{q}, \boldsymbol{\nu})+\phi_{\mathrm{m}}(t) \\
& =\left(\boldsymbol{\varphi}_{\mathrm{K}}+\boldsymbol{\varphi}_{\mathrm{s}}\right) \cdot \boldsymbol{\nu}+\phi_{\mathrm{m}} \\
& =\boldsymbol{\varphi}_{\mathrm{i}} \cdot \boldsymbol{\nu}+\phi_{\mathrm{m}}
\end{aligned}
$$

where $\phi_{\mathrm{K}}$ is Kirchhoff's potential accounting for flow due to body motion through an unbounded fluid, $\phi_{\mathrm{s}}$ is the free surface potential accounting for the modifications to the vessel-induced fluid motion due to the presence of the free surface, and $\phi_{\mathrm{m}}$ accounts for memory effects. The inner products of the normalized potential vectors $\varphi_{\mathrm{K}}$ and $\varphi_{\mathrm{S}}$ with the quasivelocity vector $\nu$ yield $\phi_{\mathrm{K}}$ and $\phi_{\mathrm{s}}$, respectively. In addition, the sum of $\phi_{\mathrm{K}}$ and $\phi_{\mathrm{s}}$ is equal to the impulsive component $\phi_{\mathrm{i}}$ of the velocity potential $\phi$ defined in Section III-A2. Therefore, the sum of the normalized potential vectors $\varphi_{\mathrm{K}}$ and $\varphi_{\mathrm{S}}$ is equal to the normalized potential vector $\varphi_{\mathrm{i}}$ for impulsive flow.

Applying the decomposed form of the potential in (18) to the reformulated LNMS model (10) yields the following equations of motion in vector/matrix form, a form motivated by robot dynamic formulations that are helpful in recognizing and exploiting physical system properties [42]

$$
\begin{aligned}
\boldsymbol{F}+\boldsymbol{F}_{I}= & \left(\boldsymbol{M}_{\mathrm{b}}+\boldsymbol{M}_{\mathcal{B}}+\boldsymbol{M}_{2}\right) \dot{\boldsymbol{\nu}} \\
& +\boldsymbol{G}\left(\boldsymbol{M}_{\mathrm{b}}+\boldsymbol{M}_{\mathcal{B}}+\boldsymbol{M}_{2}\right) \boldsymbol{\nu}+\boldsymbol{D} \boldsymbol{\nu}+\boldsymbol{E} .
\end{aligned}
$$

Here, $\boldsymbol{M}_{\mathrm{b}}$ and $\boldsymbol{M}_{\mathcal{B}}$ are identical to the matrices in (3) and (6), and

$$
\begin{aligned}
\boldsymbol{M}_{2}= & \rho \iint_{\mathcal{B}} \boldsymbol{b} \boldsymbol{\varphi}_{\mathrm{s}}^{T} d S \\
\boldsymbol{D}= & \rho \iint_{\mathcal{B}} \sum_{i=1}^{6} \nu_{i} \boldsymbol{b} \tilde{\nabla}_{i} \boldsymbol{\varphi}_{\mathrm{s}}^{T} d S-\rho \iint_{\mathcal{S}_{0}} \phi_{\mathrm{m}} \tilde{\nabla} \frac{\partial \boldsymbol{\varphi}_{\mathrm{i}}^{T}}{\partial n} d S \\
& +\frac{1}{2} \rho \iint_{\mathcal{S}_{0}} \sum_{i=1}^{6}\left(\frac{\partial \varphi_{\mathrm{i} i}}{\partial n} \boldsymbol{b} \frac{\partial \boldsymbol{\varphi}_{\mathrm{i}}^{T}}{\partial n}\right) \boldsymbol{\nu} \boldsymbol{e}_{i}^{T} d S \\
\boldsymbol{E}= & \rho \iint_{\mathcal{B}} \frac{\partial \phi_{\mathrm{m}}}{\partial t} \boldsymbol{b} d S+\rho \boldsymbol{G} \iint_{\mathcal{B}} \phi_{\mathrm{m}} \boldsymbol{b} d S \\
& -\rho \iint_{\mathcal{S}_{0}} \phi_{\mathrm{m}} \hat{\nabla} \frac{\partial \phi_{\mathrm{m}}}{\partial n} d S+\frac{1}{2} \rho \iint_{\mathcal{S}_{0}}\left(\nabla \phi_{\mathrm{i}} \cdot \nabla \phi_{\mathrm{m}}\right. \\
& \left.+\nabla \phi_{\mathrm{m}} \cdot \nabla \phi_{\mathrm{i}}+\nabla \phi_{\mathrm{m}} \cdot \nabla \phi_{\mathrm{m}}\right) \boldsymbol{b} d S .
\end{aligned}
$$

A detailed derivation of each term is provided in Appendix C. The right-hand side of (19) is equivalent to the LNM model in [1]; each term has a corresponding term in the other formulation. The physical insight and useful properties described in [1] for the LNM model, therefore, carry over to the model in (19).

\section{B. Determination of Impulsive and Memory Terms}

Here, we describe procedures for determining the impulsive and memory terms in the reformulated LNMS model in (19). The normalized potential vectors $\varphi_{\mathrm{K}}$ and $\varphi_{\mathrm{s}}$ and their derivatives can be computed by solving for the flow corresponding to impulsive motion of the body using a potential-flow panel code. In addition, the memory potential $\phi_{\mathrm{m}}$ and its derivatives can be approximated using the impulse response of the system. 
1) Computation of Impulsive Terms: As described in Section IV, the $\boldsymbol{q}$-dependent normalized potential vector for impulsive flow $\varphi_{\mathrm{i}}$ can be computed by solving for the instantaneous response of the fluid due to an impulsive motion of the body using a potential flow panel code. On the other hand, Kirchhoff's normalized potential vector $\varphi_{\mathrm{K}}$ can be obtained by considering a moving body in an unbounded fluid, independent of $\boldsymbol{q}$. In addition, the $\boldsymbol{q}$-dependent $\varphi_{\mathrm{S}}$ can be computed by subtracting $\varphi_{\mathrm{K}}$ from $\varphi_{\mathrm{i}}$. The derivatives of $\varphi_{\mathrm{K}}$ and $\varphi_{\mathrm{s}}$ can be obtained through the same procedure. Finally, by computing a set of impulsive terms in the operating range of the system, a look-up table can be created to form a discrete parameter model, or a data fit can be performed on the $\boldsymbol{q}$-dependent impulsive terms to yield a continuously parameterized model.

2) Approximation of Memory Terms: The memory potential $\phi_{\mathrm{m}}$ is the sum of a diffraction potential $\phi_{\mathrm{D}}$ and a radiation potential $\phi_{\mathrm{R}}$. Based on the derivation introduced by Falnes [43], the diffraction potential can be expressed as the following convolution integral:

$$
\phi_{\mathrm{D}}(t)=\int_{-\infty}^{\infty} h(\boldsymbol{q}(\tau), t-\tau) \eta(\tau) d \tau
$$

where $h$ is the impulse response to the wave elevation $\eta$. In addition, the radiation potential can be expressed as the following convolution integral described by Battista [2]:

$$
\phi_{\mathrm{R}}(t)=\int_{0}^{t} \boldsymbol{\varphi}_{\mathrm{R}}(\boldsymbol{q}(\tau), t-\tau) \cdot \boldsymbol{\nu}(\tau) d \tau
$$

Here, the normalized potential vector $\varphi_{\mathrm{R}}$ is the impulse response to the body's velocity $\boldsymbol{\nu}$. A set of $\boldsymbol{q}$-dependent impulse response functions $h$ and $\varphi_{\mathrm{R}}$ can be obtained in the operating range of the system using a potential-flow panel code, and the resulting sets can then be used to create a look-up table or to form a data fit. The resulting discrete set of impulse response functions, or the continuous, fitted impulse response function, can be used to approximate the convolution integrals in (20) and (21) using finite-dimensional state equations according to a method proposed by Kung [44]. That method has been implemented by several authors, including Yu and Falnes [45] and Damaren [46] for the diffraction problem and Kristiansen and Egeland [47], Taghipour et al. [48], Perez and Fossen [49], and Chen et al. [50] for the radiation problem. The same procedure can be applied to approximate the derivatives of $\phi_{\mathrm{D}}$ and $\phi_{\mathrm{R}}$ to obtain the derivatives of $\phi_{\mathrm{m}}$ in vector $\boldsymbol{E}$ of (19).

To summarize, applying the decomposed form of the velocity potential (see Section VI-A) to the reformulated LNMS model (10) produces expressions that may provide more physical insight concerning submerged vessel motion [1], [2]. Computing the impulsive terms (see Section VI-B1) and replacing the memory terms (see Section VI-B2) in the final form of the reformulated LNMS model (19) yields a model formulation well suited for control design and simulation.

\section{CONCLUSIONS AND FUTURE WORK}

In this article, incident wave effects were incorporated into a reformulated LNM model for a submerged vessel moving near a free surface to form a reformulated LNMS model. To begin, the LNM model derived by Battista [1], [2] using a Lagrangian mechanical system formulation was introduced. A reformulation of the LNM model was described, which enables parameter computation using data from a potential-flow panel code. Incident wave effects were then incorporated into the reformulated model to yield a reformulated LNMS model. The reformulated LNMS model, whose parameters can be computed using the methods described in this study, was used to predict hydrodynamic forces on a prolate spheroid undergoing sinusoidal motion in the vertical plane, and the results were compared with forces obtained from the medium-fidelity potential-flow panel code. In addition, hydrodynamic force predictions using the reformulated LNMS model were compared with panel code solutions for a prolate spheroid approaching the surface in calm water and in plane progressive waves. Finally, a control-oriented formulation of the reformulated LNMS model was proposed.

Steady surge force, heave force, and pitch moment predictions for a prolate spheroid moving at a constant forward velocity parallel to the free surface were derived from a reformulated LNM model (8) and compared to basic panel code solutions in [10] and [28]. In this study, additional terms were included in the model to account for unsteady flow due to longitudinal motions and maneuvers. In addition, incident wave forces were incorporated as external forcing to yield the reformulated LNMS model. The results for hydrodynamic force calculations in the vertical plane using the reformulated LNMS model agree well with force values output by the panel code for zero-mean, sinusoidal motions, and a surface approach maneuver. In addition, some results were shown to demonstrate sensitivity of the vertical force to changes in panel area on the undisturbed free surface. The effects of plane progressive waves on a submerged vessel undergoing a near-surface maneuver were also investigated. Finally, a control-oriented variant of the reformulated LNMS model was presented, which features a physically intuitive decomposition of the velocity potential.

Based on potential-flow theory, the reformulated LNMS model can accurately predict hydrodynamic forces and moments under ideal flow assumptions, but viscous-flow effects that play a significant role should also be incorporated into the model and the resulting, amended model should be validated against experimental data. However, for the intended application of model-based control and estimation, accuracy of the amended model is not a paramount concern; well-designed model-based feedback can compensate for modeling errors and uncertainties. The LNMS model is amenable to established nonlinear control and observer design methods. Having established a procedure to compute model parameters, ongoing work is focused on developing and testing model-based nonlinear control and estimation strategies. 


\section{APPENDIX A}

TIME-DERIVATIVE TERMS IN THE REFORMULATED LNM MODEL

With regard to the first term in the second row of (7), although the body surface $\mathcal{B}$ is in motion, the limits of the integral and the velocity potential in the integrand use the same time and spatial coordinates, as computed by the potential-flow code Aegir [40]. Therefore, the order of the derivative and the integral can simply be interchanged as shown in the following:

$$
\frac{d}{d t} \iint_{\mathcal{B}} \phi \boldsymbol{b} d S=\iint_{\mathcal{B}} \frac{\partial \phi}{\partial t} \boldsymbol{b} d S
$$

As for the second term in the second row of (7), the undisturbed free surface is fixed, so the order of the derivative and the integral can simply be interchanged. Therefore

$$
\frac{d}{d t} \iint_{\mathcal{S}_{0}} \phi \frac{\partial \varphi_{\mathrm{i}}}{\partial n} d S=\iint_{\mathcal{S}_{0}}\left[\frac{\partial \phi}{\partial t} \frac{\partial \varphi_{\mathrm{i}}}{\partial n}+\phi \frac{\partial}{\partial t}\left(\frac{\partial \varphi_{\mathrm{i}}}{\partial n}\right)\right] d S .
$$

Here, the first term in the integral can be removed since $\partial \phi / \partial t$ can be approximated as zero at the undisturbed free surface; see Appendix A-A.

With respect to the second term in the integral of (23), the normalized potential vector $\varphi_{i}$, which is expressed in the bodyfixed frame, can be expressed in the earth-fixed frame as $\varphi_{\mathcal{S}_{0} \mathrm{i}}$ using the following relation:

$$
\boldsymbol{\varphi}_{\mathrm{i}}=\boldsymbol{J}^{T} \boldsymbol{\varphi}_{\mathcal{S}_{0} \mathrm{i}}
$$

since

$$
\begin{aligned}
\phi_{\mathrm{i}} & =\boldsymbol{\varphi}_{\mathrm{i}}^{T} \boldsymbol{\nu} \\
& =\boldsymbol{\varphi}_{\mathcal{S}_{0} \mathrm{i}}^{T} \dot{\boldsymbol{q}} \\
& =\boldsymbol{\varphi}_{\mathrm{i}}^{T} \boldsymbol{J}^{-1} \boldsymbol{J} \boldsymbol{\nu} .
\end{aligned}
$$

Now, if the translational and rotational components of the normalized potential vector are considered individually

$$
\varphi_{\mathrm{i}}=\left(\begin{array}{c}
\varphi_{\mathrm{it}} \\
\varphi_{\mathrm{ir}}
\end{array}\right)
$$

The time derivative of the translational component is

$$
\begin{aligned}
\frac{\partial \boldsymbol{\varphi}_{\mathrm{it}}}{\partial t} & =\frac{\partial\left(\boldsymbol{R}^{T} \boldsymbol{\varphi}_{\mathcal{S}_{0} \mathrm{it}}\right)}{\partial t} \\
& =\hat{\boldsymbol{\omega}}^{T} \boldsymbol{R}^{T} \boldsymbol{\varphi}_{\mathcal{S}_{0} \mathrm{it}} \\
& =-\hat{\boldsymbol{\omega}} \boldsymbol{\varphi}_{\mathrm{it}} \\
& =-\boldsymbol{\omega} \times \boldsymbol{\varphi}_{\mathrm{it}} .
\end{aligned}
$$

Here, $\partial \boldsymbol{\varphi}_{\mathcal{S}_{0} \text { it }} / \partial t=\mathbf{0}$ (see Appendix A-A) for an arbitrary $\dot{\boldsymbol{q}}$. Therefore

$$
\frac{\partial}{\partial t}\left(\frac{\partial \boldsymbol{\varphi}_{\mathrm{it}}}{\partial n}\right)=-\boldsymbol{\omega} \times \frac{\partial \boldsymbol{\varphi}_{\mathrm{it}}}{\partial n}
$$

Care is required when considering the rotational component in (24). Following the procedure in [10] and [28], the rotational component can be expressed as

$$
\varphi_{\mathrm{ir}}=\frac{\partial \phi}{\partial \boldsymbol{\omega}}
$$

$$
=\boldsymbol{r} \times \frac{\partial \phi}{\partial \boldsymbol{v}}
$$

where $r$ in the integral over $\mathcal{S}_{0}$ is the position of a point on the undisturbed free surface $\mathcal{S}_{0}$ in the body-fixed frame. If the position of a point on $\mathcal{S}_{0}$ in the earth-fixed frame is denoted as $\boldsymbol{r}_{\mathcal{S}_{0}}$, then

$$
\boldsymbol{r}_{\mathcal{S}_{0}}=R \boldsymbol{r}+\boldsymbol{x}_{\mathrm{b}}
$$

Taking the time derivative

$$
\dot{\boldsymbol{r}}_{\mathcal{S}_{0}}=\dot{R} \boldsymbol{r}+R \dot{\boldsymbol{r}}+\dot{\boldsymbol{x}}_{\mathrm{b}} .
$$

Since $\boldsymbol{r}_{\mathcal{S}_{0}}$ is constant, $\dot{\boldsymbol{r}}_{\mathcal{S}_{0}}=0$. In addition, some of the terms earlier can be replaced with ones shown ahead [2]

$$
\begin{aligned}
\dot{\boldsymbol{x}}_{\mathrm{b}} & =R \boldsymbol{v} \\
\dot{R} & =R \hat{\boldsymbol{\omega}} .
\end{aligned}
$$

Therefore

$$
\dot{r}=-\boldsymbol{v}-\omega \times \boldsymbol{r} .
$$

With respect to the rotational component of the second term in the integral of (23), by applying (25)-(27)

$$
\begin{aligned}
\frac{\partial}{\partial t}\left(\frac{\partial \boldsymbol{\varphi}_{\mathrm{ir}}}{\partial n}\right) & =\frac{\partial}{\partial t}\left(\boldsymbol{r} \times \frac{\partial \boldsymbol{\varphi}_{\mathrm{it}}}{\partial n}\right) \\
& =(-\boldsymbol{v}-\boldsymbol{\omega} \times \boldsymbol{r}) \times \frac{\partial \boldsymbol{\varphi}_{\mathrm{it}}}{\partial n}+\boldsymbol{r} \times \frac{\partial}{\partial t}\left(\frac{\partial \boldsymbol{\varphi}_{\mathrm{it}}}{\partial n}\right) \\
& =(-\boldsymbol{v}-\boldsymbol{\omega} \times \boldsymbol{r}) \times \frac{\partial \boldsymbol{\varphi}_{\mathrm{it}}}{\partial n}-\boldsymbol{r} \times\left(\boldsymbol{\omega} \times \frac{\partial \boldsymbol{\varphi}_{\mathrm{it}}}{\partial n}\right) .
\end{aligned}
$$

Applying (22), (25), and (28) to the second term in the second row of (7) yields

$$
\begin{aligned}
& -\rho \frac{d}{d t} \iint_{\mathcal{S}_{0}} \phi \frac{\partial \varphi_{\mathrm{i}}}{\partial n} d S
\end{aligned}
$$

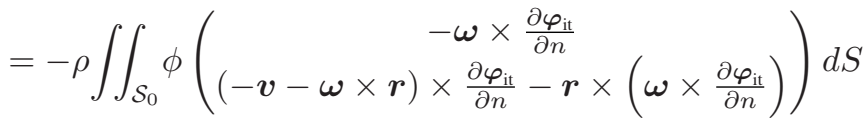

$$
\begin{aligned}
& =-\rho \iint_{\mathcal{S}_{0}} \phi\left(\begin{array}{c}
-\boldsymbol{\omega} \times \frac{\partial \boldsymbol{\varphi}_{\mathrm{it}}}{\partial n} \\
-\boldsymbol{v} \times \frac{\partial \boldsymbol{\varphi}_{\mathrm{it}}}{\partial n}-\boldsymbol{\omega} \times \frac{\partial \boldsymbol{\varphi}_{\mathrm{ir}}}{\partial n}
\end{array}\right) d S .
\end{aligned}
$$

\section{A. Linearization of $\partial \phi / \partial t$}

Recall the unsteady Bernoulli equation

$$
-\frac{1}{\rho}\left(p-p_{\mathrm{a}}\right)=\frac{\partial \phi}{\partial t}+\frac{1}{2} \nabla \phi \cdot \nabla \phi-g z .
$$

Reorganizing the unsteady Bernoulli equation as an expression of $\partial \phi / \partial t$ at the exact free surface gives

$$
\frac{\partial \phi(z)}{\partial t}=\left.\left(g z-\frac{1}{2} \nabla \phi \cdot \nabla \phi\right)\right|_{-z=\eta} .
$$

This expression is linearized about $z=0$ using its Taylor series expansion

$$
\frac{\partial \phi(z)}{\partial t}=\left.\left(g z-\frac{1}{2} \nabla \phi \cdot \nabla \phi\right)\right|_{z=0}
$$




$$
\begin{aligned}
& +\left.\frac{\partial}{\partial z}\left(g z-\frac{1}{2} \nabla \phi \cdot \nabla \phi\right)\right|_{z=0} z+\cdots \\
= & g z+\text { (higher order terms }) \\
\approx & g z .
\end{aligned}
$$

Therefore

$$
\begin{gathered}
\qquad\left.\frac{\partial \phi}{\partial t}\right|_{z=0}=0 \\
\text { APPENDIX B } \\
\text { CANCELING OUT EQUIVALENT TERMS } \\
\text { The second term in the third row of (7) can be rewritten as } \\
-\rho \boldsymbol{G} \iint_{\mathcal{S}_{0}} \phi \frac{\partial \boldsymbol{\varphi}_{\mathrm{i}}}{\partial n} d S=-\rho\left(\begin{array}{cc}
\hat{\boldsymbol{\omega}} & \mathbf{0} \\
\hat{\boldsymbol{v}} & \hat{\boldsymbol{\omega}}
\end{array}\right) \iint_{\mathcal{S}_{0}} \phi \frac{\partial \boldsymbol{\varphi}_{\mathrm{i}}}{\partial n} d S \\
=-\rho \iint_{\mathcal{S}_{0}} \phi\left(\begin{array}{cc}
\boldsymbol{\omega} \times \frac{\partial \boldsymbol{\varphi}_{\mathrm{it}}}{\partial n} \\
\boldsymbol{v} \times \frac{\partial \boldsymbol{\varphi}_{\mathrm{it}}}{\partial n}+\boldsymbol{\omega} \times \frac{\partial \boldsymbol{\varphi}_{\mathrm{ir}}}{\partial n}
\end{array}\right) d S .
\end{gathered}
$$

\section{CANCELING OUt EQUivalent TERMS}

Now, the integrals over $\mathcal{S}_{0}$ in (29) and (30) cancel each other out in (7), and the resulting reformulated LNM model can now be expressed as

$$
\begin{aligned}
\boldsymbol{F}= & \boldsymbol{M}_{\mathrm{b}} \dot{\boldsymbol{\nu}}+\boldsymbol{G} \boldsymbol{M}_{\mathrm{b}} \boldsymbol{\nu}+\rho \iint_{\mathcal{B}} \frac{\partial \phi}{\partial t} \boldsymbol{b} d S+\rho \boldsymbol{G} \iint_{\mathcal{B}} \phi \boldsymbol{b} d S \\
& -\rho \iint_{\mathcal{S}_{0}} \phi \tilde{\nabla}\left(\frac{\partial \phi}{\partial n}\right) d S+\frac{1}{2} \rho \iint_{\mathcal{S}_{0}}(\nabla \phi \cdot \nabla \phi) \boldsymbol{b} d S
\end{aligned}
$$

where

$$
\begin{aligned}
& \boldsymbol{G}=\left(\begin{array}{cc}
\hat{\boldsymbol{\omega}} & \mathbf{0} \\
\hat{\boldsymbol{v}} & \hat{\boldsymbol{\omega}}
\end{array}\right) \\
& \tilde{\nabla}=\left(\begin{array}{c}
\nabla \\
\boldsymbol{r} \times \nabla
\end{array}\right) .
\end{aligned}
$$

\section{APPENDIX C}

\section{IMPLEMENTATION OF DECOMPOSED VELOCITY POTENTIAL}

By applying the decomposition of the total velocity potential in (18), each term in the reformulated LNMS model (10) can be rewritten as

$$
\begin{aligned}
& \rho \iint_{\mathcal{B}} \frac{\partial \phi}{\partial t} \boldsymbol{b} d S \\
& =\rho \iint_{\mathcal{B}} \frac{\partial}{\partial t}\left[\left(\boldsymbol{\varphi}_{\mathrm{K}}+\boldsymbol{\varphi}_{\mathrm{s}}\right) \cdot \boldsymbol{\nu}+\phi_{\mathrm{m}}\right] \boldsymbol{b} d S \\
& =\rho \iint_{\mathcal{B}}\left[\left(\frac{\partial \boldsymbol{\varphi}_{\mathrm{s}}}{\partial t} \cdot \boldsymbol{\nu}\right) \boldsymbol{b}+\left(\boldsymbol{\varphi}_{\mathrm{i}} \cdot \dot{\boldsymbol{\nu}}\right) \boldsymbol{b}+\frac{\partial \phi_{\mathrm{m}}}{\partial t} \boldsymbol{b}\right] d S \\
& =\rho \iint_{\mathcal{B}}\left[\left(\left(\sum_{i=1}^{6} \nu_{i} \frac{\partial \boldsymbol{\varphi}_{\mathrm{s}}}{\partial q_{i}^{\prime}}\right) \cdot \boldsymbol{\nu}\right) \boldsymbol{b}+\left(\boldsymbol{\varphi}_{\mathrm{i}} \cdot \dot{\boldsymbol{\nu}}\right) \boldsymbol{b}+\frac{\partial \phi_{\mathrm{m}}}{\partial t} \boldsymbol{b}\right] d S \\
& =\rho \iint_{\mathcal{B}}\left[\left(\boldsymbol{b} \boldsymbol{\varphi}_{\mathrm{K}}^{T}\right) \dot{\boldsymbol{\nu}}\right.
\end{aligned}
$$

$$
\left.+\left(\boldsymbol{b} \boldsymbol{\varphi}_{\mathrm{s}}^{T}\right) \dot{\boldsymbol{\nu}}+\left(\sum_{i=1}^{6} \nu_{i} \boldsymbol{b} \tilde{\nabla}_{i} \boldsymbol{\varphi}_{\mathrm{s}}^{T}\right) \boldsymbol{\nu}+\frac{\partial \phi_{\mathrm{m}}}{\partial t} \boldsymbol{b}\right] d S
$$

$\rho \boldsymbol{G} \iint_{\mathcal{B}} \phi \boldsymbol{b} d S$

$=\rho \boldsymbol{G} \iint_{\mathcal{B}}\left[\left(\boldsymbol{\varphi}_{\mathrm{K}}+\boldsymbol{\varphi}_{\mathrm{S}}\right) \cdot \boldsymbol{\nu}+\phi_{\mathrm{m}}\right] \boldsymbol{b} d S$

$=\rho \boldsymbol{G} \iint_{\mathcal{B}}\left[\left(\boldsymbol{b} \boldsymbol{\varphi}_{\mathrm{K}}^{T}\right) \boldsymbol{\nu}+\left(\boldsymbol{b} \boldsymbol{\varphi}_{\mathrm{s}}^{T}\right) \boldsymbol{\nu}+\phi_{\mathrm{m}} \boldsymbol{b}\right] d S$

$-\rho \iint_{\mathcal{S}_{0}} \phi \tilde{\nabla}\left(\frac{\partial \phi}{\partial n}\right) d S$

$=-\rho \iint_{\mathcal{S}_{0}}\left(\boldsymbol{\varphi}_{\mathrm{i}} \cdot \boldsymbol{\nu}+\phi_{\mathrm{m}}\right) \tilde{\nabla}\left[\frac{\partial\left(\boldsymbol{\varphi}_{\mathrm{i}} \cdot \boldsymbol{\nu}+\phi_{\mathrm{m}}\right)}{\partial n}\right] d S$

$=-\rho \iint_{\mathcal{S}_{0}}\left(\phi_{\mathrm{m}} \tilde{\nabla} \frac{\partial \varphi_{\mathrm{i}}^{T}}{\partial n} \boldsymbol{\nu}+\phi_{\mathrm{m}} \tilde{\nabla} \frac{\partial \phi_{\mathrm{m}}}{\partial n}\right) d S$

$\frac{1}{2} \rho \iint_{\mathcal{S}_{0}}(\nabla \phi \cdot \nabla \phi) \boldsymbol{b} d S$

$$
\begin{gathered}
=\frac{1}{2} \rho \iint_{\mathcal{S}_{0}}\left(\nabla \phi_{\mathrm{i}} \cdot \nabla \phi_{\mathrm{i}}+\nabla \phi_{\mathrm{i}} \cdot \nabla \phi_{\mathrm{m}}+\nabla \phi_{\mathrm{m}} \cdot \nabla \phi_{\mathrm{i}}\right. \\
\left.+\nabla \phi_{\mathrm{m}} \cdot \nabla \phi_{\mathrm{m}}\right) \boldsymbol{b} d S \\
=\frac{1}{2} \rho \iint_{\mathcal{S}_{0}}\left\{\left[\sum_{i=1}^{6}\left(\frac{\partial \varphi_{\mathrm{i} i}}{\partial n} \boldsymbol{b} \frac{\partial \boldsymbol{\varphi}_{\mathrm{i}}^{T}}{\partial n}\right) \boldsymbol{\nu} \boldsymbol{e}_{i}^{T}\right] \boldsymbol{\nu}+\left(\nabla \phi_{\mathrm{i}}\right.\right. \\
\left.\left.\cdot \nabla \phi_{\mathrm{m}}+\nabla \phi_{\mathrm{m}} \cdot \nabla \phi_{\mathrm{i}}+\nabla \phi_{\mathrm{m}} \cdot \nabla \phi_{\mathrm{m}}\right) \boldsymbol{b}\right\} d S .
\end{gathered}
$$

Therefore, the resulting equations of motion can be expressed as

$$
\begin{aligned}
\boldsymbol{F}+\boldsymbol{F}_{I}= & \left(\boldsymbol{M}_{\mathrm{b}}+\rho \iint_{\mathcal{B}} \boldsymbol{b} \boldsymbol{\varphi}_{\mathrm{K}}^{T} d S+\rho \iint_{\mathcal{B}} \boldsymbol{b} \boldsymbol{\varphi}_{\mathrm{s}}^{T} d S\right) \dot{\boldsymbol{\nu}} \\
& +\boldsymbol{G}\left(\boldsymbol{M}_{\mathrm{b}}+\rho \iint_{\mathcal{B}} \boldsymbol{b} \boldsymbol{\varphi}_{\mathrm{K}}^{T} d S+\rho \iint_{\mathcal{B}} \boldsymbol{b} \boldsymbol{\varphi}_{\mathrm{s}}^{T} d S\right) \boldsymbol{\nu} \\
& +\left(\rho \iint_{\mathcal{B}} \sum_{i=1}^{6} \nu_{i} \boldsymbol{b} \tilde{\nabla}_{i} \boldsymbol{\varphi}_{\mathrm{s}}^{T} d S-\rho \iint_{\mathcal{S}_{0}} \phi_{\mathrm{m}} \tilde{\nabla} \frac{\partial \boldsymbol{\varphi}_{\mathrm{i}}^{T}}{\partial n} d S\right. \\
& \left.+\frac{1}{2} \rho \iint_{\mathcal{S}_{0}} \sum_{i=1}^{6}\left(\frac{\partial \varphi_{\mathrm{i} i}}{\partial n} \boldsymbol{b} \frac{\partial \boldsymbol{\varphi}_{\mathrm{i}}^{T}}{\partial n}\right) \boldsymbol{\nu} \boldsymbol{e}_{i}^{T} d S\right) \boldsymbol{\nu} \\
& +\rho \iint_{\mathcal{B}} \frac{\partial \phi_{\mathrm{m}}}{\partial t} \boldsymbol{b} d S+\rho \boldsymbol{G} \iint_{\mathcal{B}} \phi_{\mathrm{m}} \boldsymbol{b} d S \\
& -\rho \iint_{\mathcal{S}_{0}} \phi_{\mathrm{m}} \hat{\nabla} \frac{\partial \phi_{\mathrm{m}}}{\partial n} d S \\
& +\frac{1}{2} \rho \iint_{\mathcal{S}_{0}}\left(\nabla \phi_{\mathrm{i}} \cdot \nabla \phi_{\mathrm{m}}+\nabla \phi_{\mathrm{m}} \cdot \nabla \phi_{\mathrm{i}}\right. \\
& \left.+\nabla \phi_{\mathrm{m}} \cdot \nabla \phi_{\mathrm{m}}\right) \boldsymbol{b} d S .
\end{aligned}
$$




\section{ACKNOWLEDGMENT}

The authors would like to thank Navatek, Ltd., for providing the seakeeping code Aegir, as well as technical support. The authors would also like to thank Drs. F. Valentinis, T. Battista, and E. G. Paterson for comments that helped improve and clarify this article.

\section{REFERENCES}

[1] T. Battista, F. Valentinis, and C. Woolsey, "A maneuvering model for an underwater vehicle near a free surface-Part I: Motion without memory effects," IEEE J. Ocean. Eng., vol. 45, no. 1, pp. 212-226, Jan. 2020.

[2] T. Battista, "Lagrangian mechanics modeling of free surface-affected marine craft," Aerospace and Ocean Eng., Ph.D. dissertation, Dept. Aerospace Ocean Eng., Virginia Tech, Blacksburg, VA, USA, 2018.

[3] C. Crane, H. Eda, and A. Landsburg, "Controllability," in Principles of Naval Architecture. Jersey City, NY, USA: SNAME, vol. 3, 1989, ch. 9, pp. 191-429.

[4] H. Lamb, Hydrodynamics. New York, NY, USA: Dover, 1932.

[5] M. Abkowitz, "Lectures on ship hydrodynamics-steering and manoeuvrability," Tech. Rep. Hy-5, Hydro-Aerodyn. Lab., Lyngby, Denmark, 1964.

[6] N. Norrbin, "Theory and observations on the use of a mathematical model for ship manoeuvring in deep and confined waters," Tech. Rep. SSPA-Pub68, Swedish State Shipbuilding Exp. Tank, Goteborg, Sweden, 1971.

[7] K. Son and K. Nomoto, "On the coupled motion of steering and rolling of a high-speed container ship," J. Soc. Nav. Architects Jpn., vol. 150, pp. 232-244, 1981.

[8] A. Christensen and M. Blanke, "A linearized state-space model of steering and roll of a high-speed container ship," Tech. Rep. 86-D-574, Tech. Univ. Denmark, Lyngby, Denmark, 1986.

[9] T. Fossen, Guidance and Control of Ocean Vehicles. Hoboken, NJ, USA: Wiley, 1994.

[10] S. Jung, S. Brizzolara, and C. Woolsey, "Parameter computation for a Lagrangian mechanical system model of a submerged vessel moving near a free surface," Ocean Eng., to be published.

[11] T. Battista, S. Jung, C. Woolsey, and E. Paterson, "An energy-casimir approach to underwater vehicle depth and heading regulation in short crested waves," in Proc. IEEE Conf. Control Technol. Appl., vol. 45, 2017, pp. 217-222.

[12] G. Weinblum and M. St. Denis, "On the motions of ships at sea," Trans. SNAME, vol. 58, pp. 184-231, 1950.

[13] R. Beck, W. Cummins, J. Dalzell, P. Mandel, and W. Webster, "Motions in waves," in Principles of Naval Architecture. Jersey City, NY, USA: SNAME, vol. 3, 1989, ch. 8, pp. 1-190.

[14] J. Newman, "Theory of ship motions," in Proc. Adv. Appl. Mech., vol. 18, 1978, pp. 221-283.

[15] D. C. Kring, Time domain ship motions by a three-dimensional Rankine panel method," Ocean Eng., Ph.D. dissertation, Dept. Ocean Eng., MIT, Cambridge, MA, USA, 1994.

[16] T. Crook, "An initial assessment of free surface effects on submerged bodies," Master's thesis, Nav. Postgrad. Schl., Monterey, CA, USA, Sep. 1994.

[17] P. Montgomery and P. Crossland, "User guide for the PAT-95 suite of ship motion computer programs," MoD Rep. DRA/SS/SSHE/CR95030, 1995.

[18] T. Gourlay, A. Graefe, V. Shigunov, and E. Lataire, "Comparison of AQWA, GL RANKINE, MOSES, OCTOPUS, PDStrip and WAMIT with model test results for cargo ship wave-induced motions in shallow water," in Proc. ASME 34th Int. Conf. Ocean, Offshore, Arctic Eng., 2015, pp. $1-10$.

[19] "ShipMo3D version 3.0 user manual for computing ship motions in the time and frequency domains," Tech. Memo. TM 2011-308, DRDC, Dartmouth, NS, Canada, 2012.

[20] Navatek, "Aegir user guide," Tech. Rep., Navatek Ltd., Newport, RI, USA, 2018.

[21] T. Ogilvie, "Singular-perturbation problems in ship hydrodynamics," Adv. Appl. Mech., vol. 17, pp. 91-188, 1977.

[22] O. Grim, "A method for a more precise computation of heaving and pitching motions both in smooth water and in waves," in Proc. 3rd Symp. Nav. Hydrodyn., 1960, pp. 483-524.

[23] H. Eda, "Directional stability and control of ships in waves," J. Ship Res., vol. 16 , pp. 205-218, 1972.
[24] P. Bailey, W. Price, and P. Temarel, "A unified mathematical model describing the manoeuvring of a ship travelling in a seaway," Trans. Roy. Inst. Nav. Architects, vol. 140, pp. 131-149, 1998, doi: 10.3940/rina.sbt.1998.a7.

[25] T. Perez, Ship Motion Control: Course Keeping and Roll Stabilisation Using Rudder and Fins. Berlin, Germany: Springer-Verlag, 2005.

[26] T. I. Fossen, "A nonlinear unified state-space model for ship maneuvering and control in a seaway," Int. J. Bifurcation Chaos, vol. 15, no. 9, pp. 2717 2746, 2005.

[27] B. Maskew and F. A. Dvorak, "Predicting dynamic separation characteristics of general configurations," Tech. Rep. AIAA 86-1813, Analytical Methods Inc., Renton, WA, USA, 1987.

[28] S. Jung, "Determining parameters for a Lagrangian mechanical system model of a submerged vessel maneuvering in waves," Aerospace and Ocean Eng., Ph.D. dissertation, Dept. Aerospace Ocean Eng., Virginia Tech, Blacksburg, VA, USA, 2020.

[29] H. K. Khalil, Nonlinear Systems, 3rd ed. Englewood Cliffs, NJ, USA Prentice-Hall, 2002.

[30] W. Rugh, Linear System Theory. Englewood Cliffs, NJ, USA: PrenticeHall, 1996.

[31] P. Dorato, C. T. Abdallah, and V. Cerone, Linear Quadratic Control: An Introduction. Malabar, FL, USA: Krieger Publishing, 1995.

[32] W.-H. Chen, J. Yang, L. Guo, and S. Li, "Disturbance-observer-based control and related methods-An overview," IEEE Trans. Ind. Electron., vol. 63, no. 2, pp. 1083-1095, Feb. 2016.

[33] J.-J. Slotine and W. Li, Applied Nonlinear Control. Englewood Cliffs, NJ, USA: Prentice-Hall, 1991.

[34] M. Krstic, I. Kanellakopoulos, and P. Kokotovic, Nonlinear and Adaptive Control Design. Hoboken, NJ, USA: Wiley, 1995.

[35] C. Woolsey, C. K. Reddy, A. M. Bloch, D. E. Chang, N. E. Leonard, and J. E. Marsden, "Controlled Lagrangian systems with gyroscopic forcing and dissipation," Eur. J. Control, vol. 10, no. 5, pp. 478-496, 2004.

[36] P. Thomasson and C. Woolsey, "Vehicle motion in currents," IEEE J. Ocean Eng., vol. 38, no. 2, pp. 226-242, Apr. 2013.

[37] C. A. Woolsey and L. Techy, "Cross-track control of a slender, underactuated AUV using potential shaping," Ocean Eng., vol. 36, no. 1, pp. 82-91, 2009, doi: 10.1016/j.oceaneng.2008.07.010.

[38] F. Valentinis, A. Donaire, and T. Perez, "Energy-based motion control of a slender hull unmanned underwater vehicle," Ocean Eng., vol. 104, no. 1, pp. 604-616, 2015.

[39] R. Datla, H. Kim, and J. Stebe, "Evaluation of a CFD Program Aegir for bare hull resistance and seakeeping prediction capability," Tech. Rep. NSWCCD-CISD-2009/010, Carderock Div., Nav. Surf. Warfare Ctr., West Bethesda, MD, USA, 2009.

[40] D. C. Kring, Aegir Theory Guide, Navatek Ltd., 2017.

[41] G. P. Weinblum, "On Hydrodynamic Masses," Tech. Rep. 809, David Taylor Model Basin, 1952.

[42] T. Fossen, Handbook of Marine Craft Hydrodynamics and Motion Control. Hoboken, NJ, USA: Wiley, 2011.

[43] J. Falnes, "On non-causal impulse response functions related to propagating water waves," Appl. Ocean Res., vol. 17, pp. 379-389, 1995, doi 10.1016/S0141-1187(96)00007-7.

[44] S. Kung, "A new identification and model reduction algorithm via singular value decompositions," in Proc. 12th Asilomar Conf. Circuits, Syst., Comput., 1978, pp. 705-714.

[45] Z. Yu and J. Falnes, "State-space modelling of a vertical cylinder in heave," Appl. Ocean Res., vol. 17, pp. 265-275, 1995, doi: 10.1016/01411187(96)00002-8

[46] C. Damaren, "A causal transient water wave diffraction formulation," Appl. Ocean Res., vol. 22, pp. 267-280, 2000, doi: 10.1016/S01411187(00)00020-1.

[47] E. Kristiansen and O. Egeland, "Frequency-dependent added mass in models for controller design for wave motion damping," IFAC Proc. Vol., vol. 36, pp. 67-72, 2003.

[48] R. Taghipour, T. Perez, and T. Moan, "Hybrid frequency-time domain models for dynamic response analysis of marine structures," Ocean Eng., vol. 35, pp. 685-705, 2008, doi: 10.1016/j.oceaneng.2007.11.002.

[49] T. Perez and T. Fossen, "A MATLAB toolbox for parametric identification of radiation-force models of ships and offshore structures," Model., Identification, Control, vol. 30, no. 1, pp. 1-15, 2009.

[50] Y. Chen, S. Jung, and C. Woolsey, "Longitudinal, near-surface maneuvering of a prolate spheroid," IFAC-PapersOnLine, vol. 52, pp. 248-253, Sep. 2019, doi: 10.1016/j.ifacol.2019.12.315. 


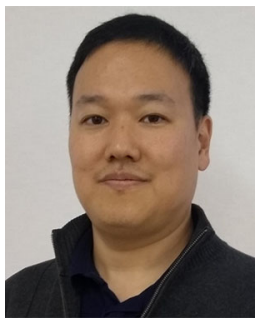

Seyong Jung (Member, IEEE) received the B.S. and M.S. degrees in mechanical engineering from Yonsei University, Seoul, South Korea, in 2003 and 2005, respectively, and the Ph.D. degree in aerospace engineering from Virginia Tech, Blacksburg, VA, USA, in 2020 .

He is currently a Senior Researcher with the New \& Renewable Energy Lab, KEPCO Research Institute, Daejeon, South Korea. His research interests include modeling and control of marine structures and vehicles, with a current focus on floating offshore wind turbines.

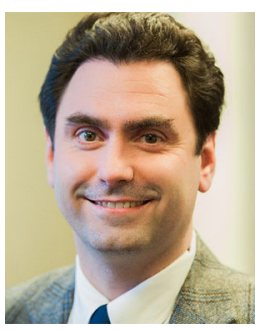

Stefano Brizzolara received the Ph.D. degree in naval architecture and marine engineering.

$\mathrm{He}$ is currently an Associate Professor with the Kevin T. Crofton Department of Aerospace and Ocean Engineering, Blacksburg, VA, USA, the Director of the VT iShip Lab, Virginia Tech, Blacksburg, and an Associate Director of the Center of Marine Autonomy and Robotics. He has been a Peabody Visiting Associate Professor with the Department of Mechanical Engineering, MIT, Cambridge, MA, USA. He is the author of more than 250 peer reviewed papers, and a designated inventor of six patents for innovative ship designs or ship systems. His research interests include computational fluid dynamics (CFD), design of advanced marine underwater and surface vehicles, design of high.]performance marine propulsors, and numerical hydrodynamics for ship design.

Dr. Brizzolara is a recipient of the 2018 Calder Prize for Best Paper on high speed crafts, the 2016 Excellence in Review for the IEEE JOURNAL OF OCEANIC ENGINEERING, and the 2015 Mandel's Prize for Excellence in Hydrofoil Research advising.

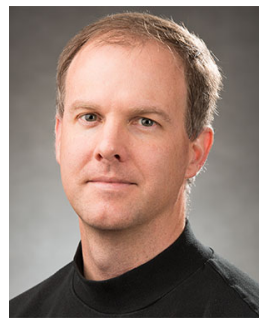

Craig Woolsey (Senior Member, IEEE) received the B.M.E. degree from Georgia Tech, Atlanta, GA, USA, in 1995, and the Ph.D. degree in mechanical and aerospace engineering from Princeton University, Princeton, NJ, USA, in 2001.

$\mathrm{He}$ is currently a Professor with the Kevin T. Crofton Department of Aerospace and Ocean Engineering, Virginia Tech, Blacksburg, VA, USA. His research and teaching focus on the modeling and control of ocean and atmospheric vehicles.

Dr. Woolsey participates in the Oceanic Engineering Society and the Control Systems Society. He is also active on the IFAC Technical Committee on Marine Systems and the AIAA Atmospheric Flight Mechanics Technical Committee. 